\title{
Beauty is in the Eye of the Cultural Beholder
}

\author{
Jiayu Zhan ${ }^{1} \quad$ Meng Liu $^{2} \quad$ Oliver G. B. Garrod ${ }^{1} \quad$ Christoph Daube ${ }^{1}$, \\ Jiayu.Zhan@glasgow.ac.uk \\ Robin A. A. Ince ${ }^{1}$ \\ Rachael E. Jack ${ }^{12}$ \\ Philippe G. Schyns ${ }^{12}$ \\ Philippe.Schyns@glasgow.ac.uk \\ ${ }^{1}$ Institute of Neuroscience and Psychology, University of Glasgow, Scotland G12 \\ 8QB, United Kingdom \\ ${ }^{2}$ School of Psychology, University of Glasgow, Scotland G12 8QB, United Kingdom
}

\begin{abstract}
Is face beauty universally perceived from a common basis of objectively definable face features, or is it irreducibly subjective and in the idiosyncratic eye of the cultural, or even individual beholder? We addressed this longstanding debate by objectively modelling the face beauty preferences of 80 individual male participants across Western European (WE) and East Asian (EA) cultures. With state-of-the-art 3D face capture technology, we derived a generative model that synthesized on each trial a random WE or EA face whose shape and complexion is constrained by natural face variations. Each participant rated the attractiveness of the face on a Likert scale. We then reverse correlated these subjective ratings with the synthesized shape and complexion face parameters to reconstruct individual face models of attractiveness for same and other ethnicity faces. By analyzing the resulting 80 individual models and reconstructing the representation space of face beauty, we addressed several key questions. Against popular belief, we show that the most attractive faces are not average face. Instead, attractive features are at the outskirts of the natural distribution of face variations, suggesting a selection pressure away from the average. Such features also form their own subspace that is separate from cues of sexual dimorphism (i.e. masculine vs. feminine). Finally, we reveal the global preferences of face features across cultures, and specific cultural and individual participant idiosyncrasies. Our results therefore represent face attractiveness in its diversity to inform and impact fundamental theories of human social perception and signalling and the design of globalized digital avatars.
\end{abstract}




\section{Introduction}

Face attractiveness confers considerable advantages in social interactions (1). A fundamental question is what makes a face attractive? To explain common preferences in a population, most theories have referred to measurable standards, such as the closeness of attractive faces to the population average (2), their bilateral symmetry (3)(4) or sexual dimorphism (5). However, mounting diverging evidence suggests that these standards do not capture facial attractiveness particularly well (6)(7)(8). One reason is that they cannot explain idiosyncratic preferences at the cultural or individual level $(9)(10)(11)(12)$. Hence, to represent and explain face attractiveness in its diversity, it is important to instead start with modelling the preferences of individuals, their culture, and then compare attractiveness preferences across cultures.

To meet these aims, we reconstructed the face feature space that represents facial attractiveness in individual cultural participants. Specifically, we objectively modelled the face features-3D shape and $L^{*} a^{*} b^{*}$ complexion-that subjectively modulated the perception of female attractiveness in each of 80 heterosexual males from two distinct cultures-i.e. 40 white Western Europeans (WE) and 40 Chinese East Asians (EA, see Materials and Methods, Participants). Our design controlled the ethnicity of the faces as a between-participant factor, with half of the cultural participants rating faces of their own ethnicity and half the other ethnicity. We did not test the perception of male attractiveness in heterosexual females, due to possible large within-individual preference variations that correlate with hormonal changes of the menstrual cycle $(13)(14)(15)(16)$, but see (17)(18).

To reconstruct the representation space of face attractiveness, our analyses extended beyond the common practice of computing group averages in experimental designs that are focused on testing the specific hypothesis of a given theory. Instead, we used a data-driven design to reverse engineer the individual subjective preferences of participants in each culture, from a rich set of naturally varying random 3D face stimuli synthesized by a linear generative model of 3D face identity (henceforth, GMF, i.e. see Materials and Method, 3D face stimuli). The GMF (19) models the shape and complexion of each face based on the sum of a categorical average component (i.e. GMF categorical average, with set controlled factors of age: 25 years old, sex: female, and ethnicity: WE or EA), plus a residual random component of parameters that controls the identity of each face. The GMF therefore captures and generates the natural variations of shape and complexion in the target population, demonstrated also by its ability to model new real faces outside the database with very low reconstruction error (see Supplementary Fig. S1C).

In the experiment, each participant saw on each of 1,950 trials a generated 3D face displayed in one of three possible viewpoints $\left(-30,0\right.$ and $+30^{\circ}$ of rotation in depth). Participants assessed the attractiveness of the face-on a 9-point rating scale from 1 , not attractive at all to 9, very attractive (see Materials and Methods, Procedure). Across all trials, we linearly regressed the variations of each GMF identity parameter of shape and complexion with the corresponding variations of the participant's attractiveness scores, to derive individual models of face attractiveness (i.e. see Materials and Methods, Analyses, Linear Regression Model). Our analyses produced a total of 80 such 
individual models (i.e. 20 models of each observer-face ethnicity combinations) that we validated (see Materials and ethods, Analyses, Model Validation).

Using these models, we tested longstanding questions about face attractiveness. First, we asked two key questions about attractive feature selection: Is face attractiveness in the face average? Are attractive face features an exaggeration of sexual dimorphism? Having shown that it is neither we reconstructed the unique feature representation space of facial attractiveness to address questions of whether such feature preferences are similar within and across cultures, and whether such cultural preferences transfer to faces of other ethnicities.

\section{Results}

\section{Feature selection: Is attractiveness in the face average?}

We can address this question simply within our linear model by computing whether (and, if so, how) the modelled face features of attractiveness depart from the average face (i.e. average 25-year old WE or EA female). For a fair comparison to the classic hypothesis that attractiveness is in the face average, we performed these analyses on the group model that averages the individual models of the same-ethnicity conditions-i.e. from 20 WE participants rating WE faces, henceforth "Western-same" and 20 EA participants rating EA faces, henceforth, "Eastern-same."

$3 D$ face shape. Figure 1 shows the group models of the Western-same and Eastern-same groups (in Figure 1A and 1B, panels 1 to 3 ). In each culture, attractive faces were systematically smaller than their respective GMF categorical average (cf. blue-coded inward deviations of the cheeks and jaw line in relation to the average shown as a grey mesh in Figure 1A and 1B). Attractive faces also had a prominent forehead and round and protruding eyes (cf. red-coded outward deviation of these features from the categorical averages in Figure 1A and 1B). However, there were also marked cultural differences, of an extruding (poutier) mouth shape in WE and a higher nose bridge and pointier chin in EA (see Figure $1 \mathrm{~A}$ and $1 \mathrm{~B}$, panels 2 and 3 for enlargement). Supplementary Fig. S2 reports each individual model, as significant inward/outward shape deviations that linearly relate to the attractiveness ratings.

Complexion. Results revealed that both cultures preferred darker than average eyelids and eyelashes (i.e. at higher contrast, see Figure $1 \mathrm{~A}$ and $1 \mathrm{~B}$, panels 4-6). WE preferred a darker (Figure 1A, panel 4) and redder (Figure 1A, panel 5) than average complexion whereas EA preferred a lighter complexion (Figure 1B, panel 4 and 6) and redder lips (Figure 1B, panel 5). Supplementary Fig. S2 reports each individual complexion model.

Note that both shape and complexion deviate in opposite directions for the unattractive features, due to linear modelling (see Materials and Methods, Analyses, Linear Regression Model). To visualize the attractive and unattractive faces in each culture, we added the same-ethnicity shape and complexion attractive deviations to the GMF 
categorical average of each ethnic female face (see Figure 1A and Figure 1B, 7-9 and Materials and ethods, Analyses, Reconstructing attractive and unattractive faces).

A. Western-same

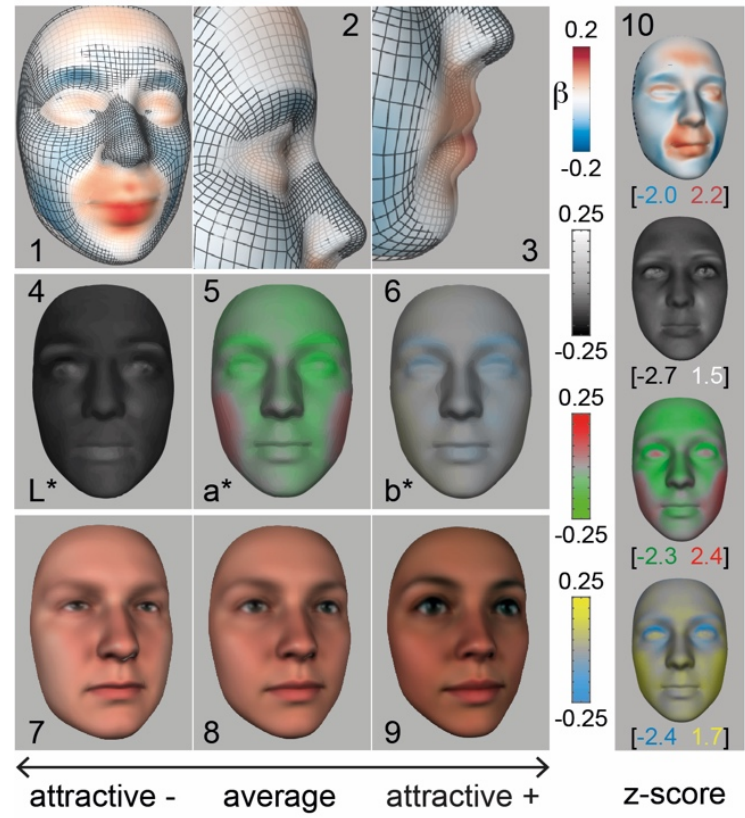

C. Western-other

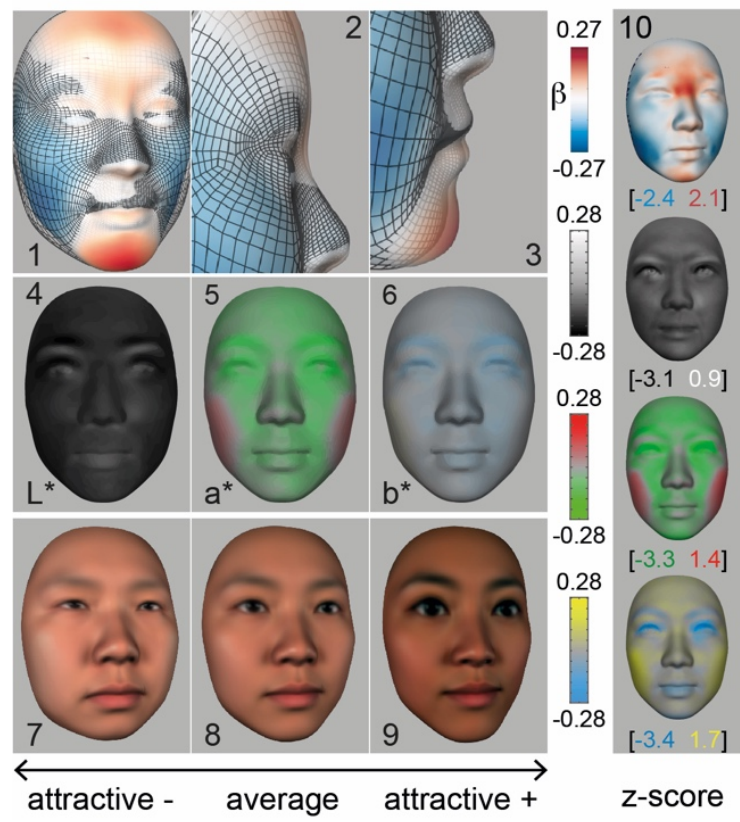

B. Eastern-same

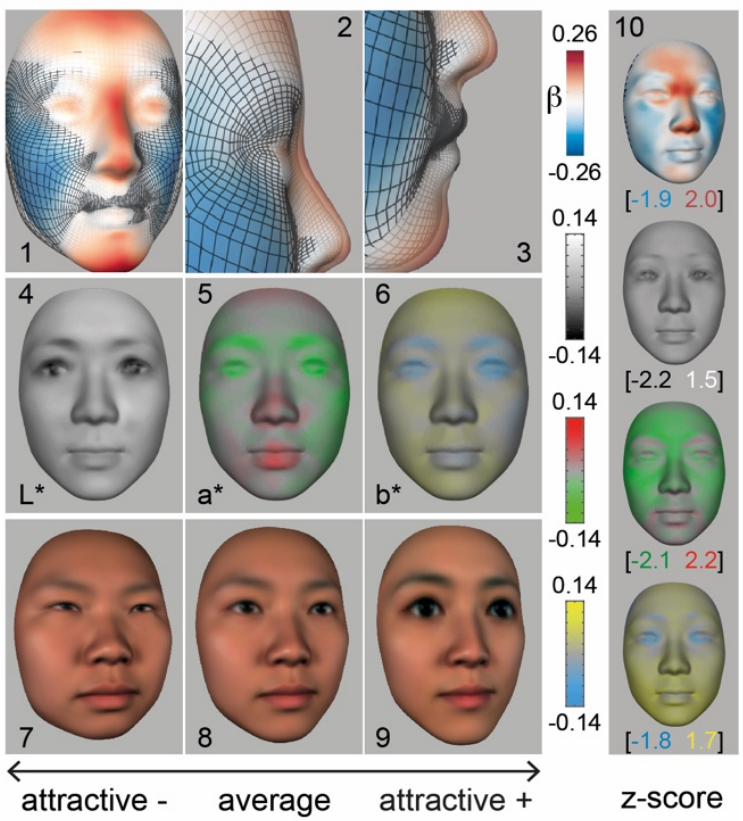

\section{Eastern-other}

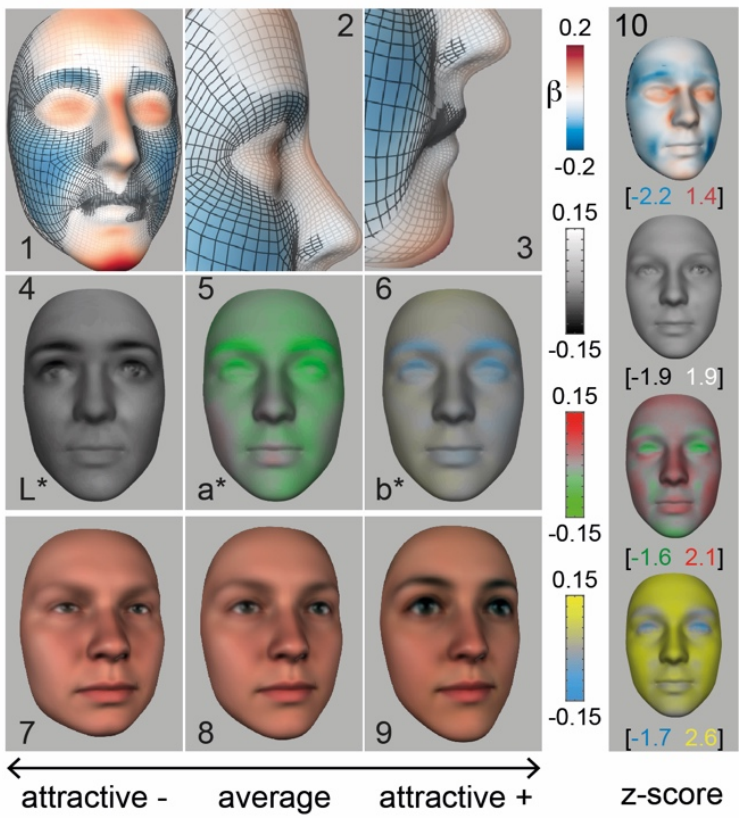

Figure 1. Female face features that modulate perceptions of attractiveness.

A. Western-same. Panel 1 shows the attractive shape features averaged across participants, with panels 2 and 3 for enlargements in profile. Attractive 3D shape features deviated both outward (in red) and inward (in blue) from the average WE female face (represented as the grey mesh). Panel 4-6 presents the attractive complexion features averaged across participants in L*a*b* color space. The corresponding color scales report the magnitude of the shape and $L^{*} a^{*} b^{*}$ 
deviations, quantified by the slope $\beta$ in the linear regression models. Panel 7 and 9 reconstruct the unattractive and attractive 3D face as shape and complexion deviations from the average WE female face (panel 8). Panel 10 shows z-scored shape and complexion deviations corresponding to the attractive faces; bracketed values show the minimal and maximal z-scores of these attractive deviations. B. Eastern-same. C. Western-other. D. Eastern-other. B, C, D are presented in a same format as in A.

\section{Feature selection: Where is attractiveness located away from the average face?}

Having shown in each culture that female face attractiveness departs from the mathematical face average, we now ask where-in terms of how distant from the average-attractive features lie in the natural distribution of female face variations. To address this, we first computed the variations of each generative face parameter in the female faces that we had showed to participants. Then, we compared the attractive deviations of highly attractive faces to this distribution (by z-scoring, see Materials and Method, Analyses, Face average and feature distribution). Figure 1 (panel 10) shows the results. We found that in both cultures, attractive shape and complexion features both sit at the outskirts of the natural distributions of variations (i.e. $>1.5$ std away from the sampling range of deviations), akin to a "hidden preference" (i.e. or peak drift, (20)(21)) in the male mechanisms of female face processing. These features comprise a pouty mouth (in WE), and the high nose bridge and pointy chin (in EA) - two face shape features that are popular in plastic surgery in each culture (22)(23), respectively. For complexion, the dark eyes reflect a general make-up choice; a darker skin in WE (24) and the lighter skin in EA (24)(25) are also consistent with women's cultural cosmetic choices. Supplementary Fig. S3 and Fig. S4 list the attractive female features reported for the WE and EA cultures in psychology studies and in cosmetic and plastic surgery reports. These closely match the attractive features of our models.

\section{Feature Selection: Is attractiveness an exaggeration of average sexual dimorphism?}

We next asked whether attractive shape and complexion features map onto features of sexual dimorphism-i.e. those that make faces look more masculine or feminine, and signalling sexual maturity and reproductive potential (26)(27)(28)? To address this, we first computed the direction of shape and complexion deviations that transform the GMF average male face into a GMF average female, separately for each ethnicity. Next, we compared this direction of sexual dimorphism (e.g., making the face more feminine) with the direction that makes the face appear more attractive, for shape and complexion (see Materials and Methods, Analysis, Sexual Dimorphism and Attractiveness). In both ethnicities, the directions of face sexual dimorphism were near orthogonal to those of face attractiveness (see Figure 2A and B). The pouty mouth, darker skin and redder cheeks preferred by Westerners and the high nose bridge, pointy chin, prominent forehead, and high contrast eye regions preferred by Easterners (denoted as solid line black arrows in Figure 2A and B) are each near orthogonal to the feminine deviations (quantified by vector cosine), represented by high cheekbones, smaller forehead and nose, and a lighter skin (denoted as dashed arrows in Figure 2). These results demonstrate that the shape and complexion deviations of face attractiveness are not exaggerations of (i.e. colinear with) feminine sexual dimorphism. Instead, facial attractiveness is an independent face evaluation (and perhaps evolution) with its own feature space. Supplementary Fig. S5 reports the distribution of attractiveness vs. sexual dimorphism similarity of individual models. 
We repeated these analyses for both Western and Eastern participants who viewed other ethnicity faces (henceforth, "Western-other" and "Eastern-other" models). Results showed replication of the main finding that attractive representations deviate from the female face average (see Figure 1, C and D) and reside in their own near-orthogonal space to sexual dimorphism (see Figure 2, C and D).

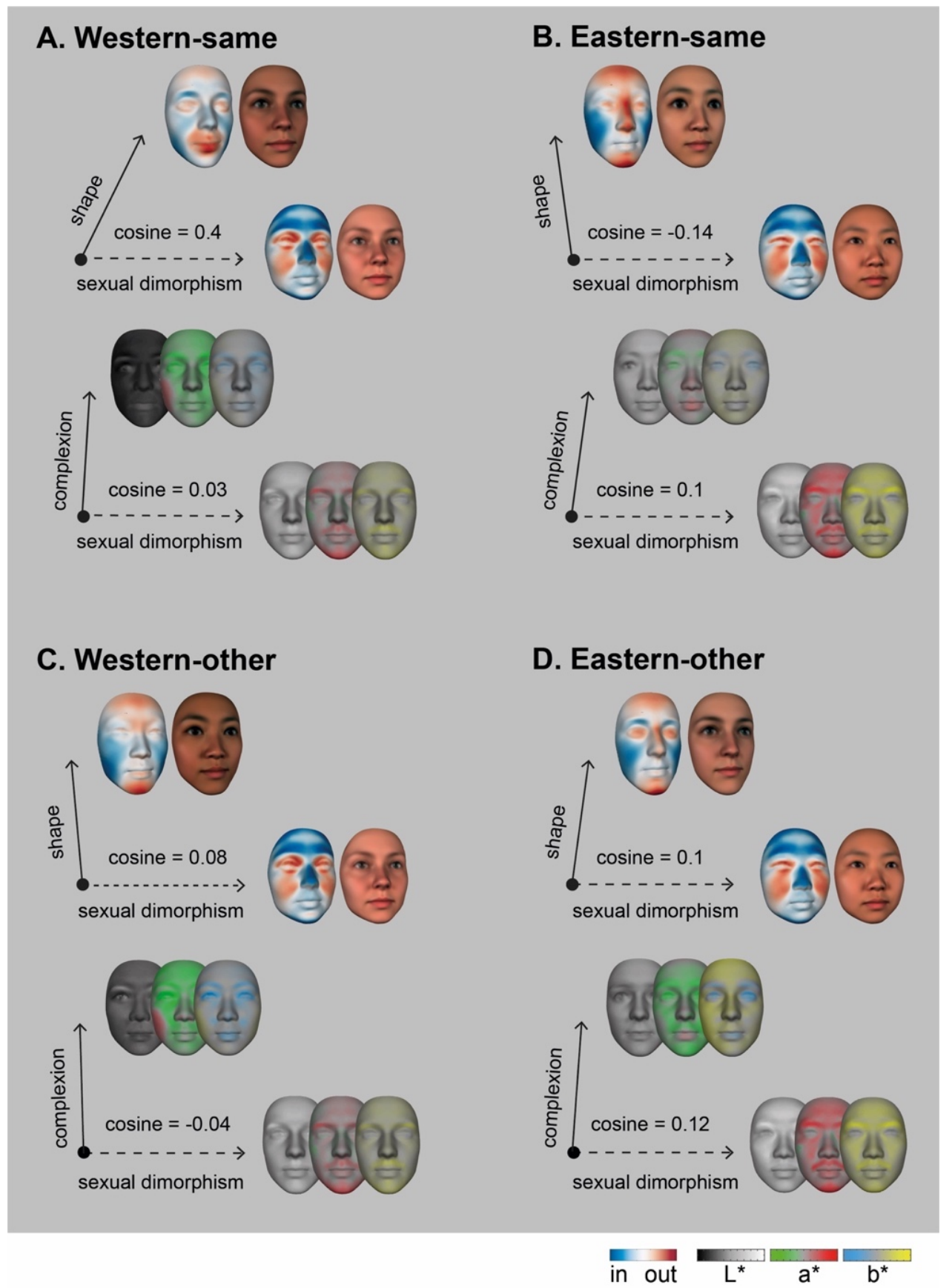

Figure 2. Face features of attractiveness and sexual dimorphism are orthogonal.

A. Western-same. In the representation space, each axis illustrates two directions of multivariate deviations from the GMF female categorical average (represented as the black dot central to both 
axes). The solid line arrow illustrates the 42-dimensional direction of attractive face shape change (top panel; 116-dimensional complexion, bottom panel) in relation to a femininized face (dashed line arrow). The vector cosine between the two multivariate axes quantifies their relationship in the representation space, where a value of 0 indicates orthogonality of shape or complexion change between attractiveness and sexual dimorphism. Faces adjacent to each axis illustrate the multivariate contents of shape and complexion changes from the average. B. Eastern-same. C. Western-other. D. Eastern-other. All face deviations are displayed on a normalized color scale (to the maximum across all displays; sexual dimorphism is amplified for display purposes; the multivariate dimensionality of shape is 42 ; complexion is 116 ).

\section{Culture: Cultural commonalities, preferences, and individual differences}

Analyses at the level of the average cultural models revealed that attractive face features deviate from the female face average, lie at the outskirts of the natural distribution, and represent changes in directions different to feminine sexual dimorphism. This suggests a space that represents face attractiveness on its own. We now model this feature space and, within it, explain commonalities across cultures, their differences, and individual differences.

The representation space of face attractiveness. To derive this space, we applied Principal Components Analysis (PCA) to all 80 individual models (i.e. Western-same and different, Eastern-same and different), separately for their shape (Figure $3 \mathrm{~A}$ ) and complexion (Figure 3B) (see Materials and Methods, Analyses, Components of attractiveness). PCA delivered a multi-dimensional feature space that represents and can explain the principal variations of attractive face features within and across cultures and their individual members. Here, the first three components captured $74 \%$ of shape variance (vs. $97 \%$ of complexion variance). Figure 3 represents these first three PCs as separate axes, where individual cultural participant models are represented as colored dots whose positions indicate a composition of the attractive features making up the space. To illustrate, in Figure 3A, the leftmost small purple dot (see purple arrow) is an individual Western-same shape (vs complexion in Figure 3B) model with representation space coordinates $-3.1,-11.3,-10.7$ on shape PC1, PC2 and PC3 (vs. -6.6, 0, 0 on complexion PCs). Each coordinate indicates the strength of representation of the corresponding PC's information contents (see small faces next to each axis) in the individual model. We next detail how we used this space of attractive components to address questions of cultural commonalities and differences, individual participant idiosyncrasies and the transfer of these preferences across face ethnicities. 

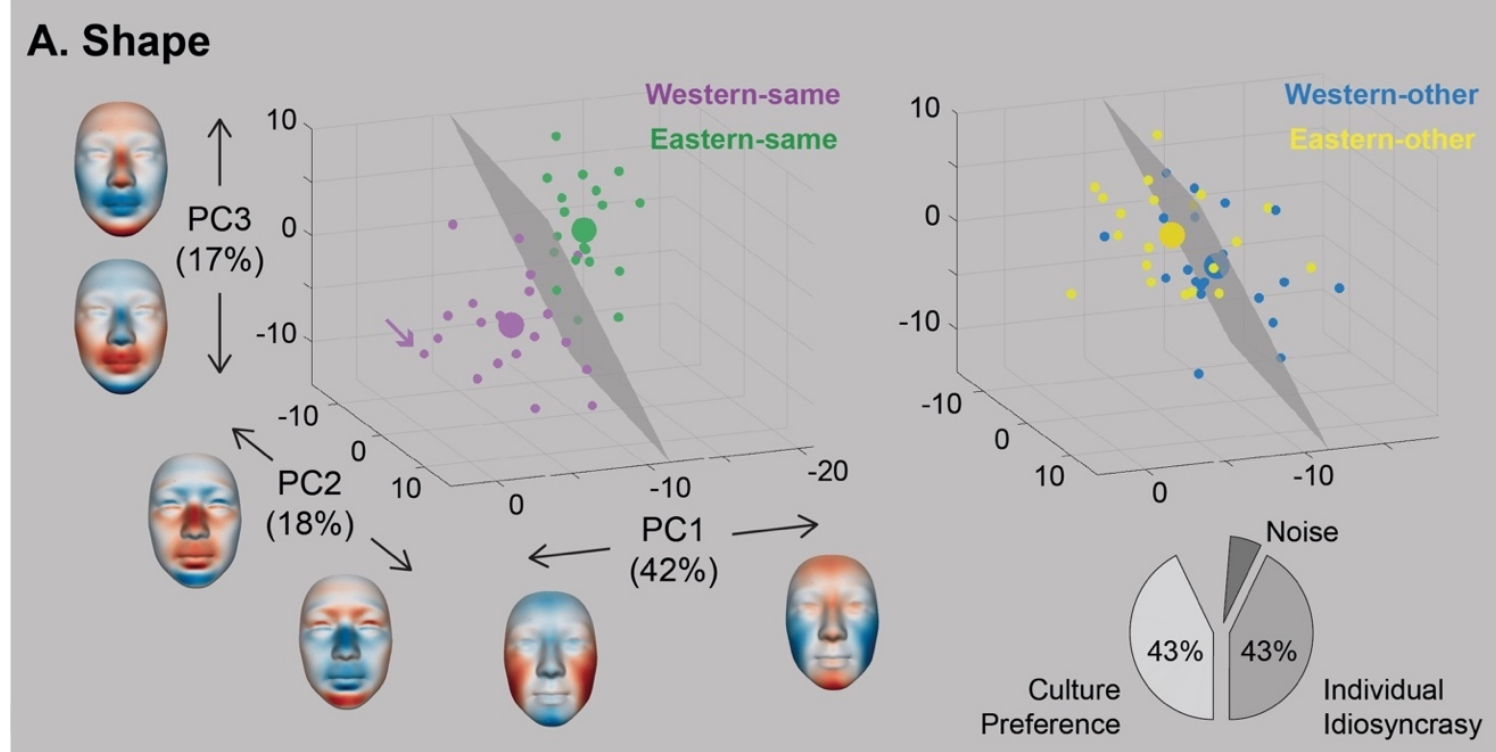

\section{B. Complexion}

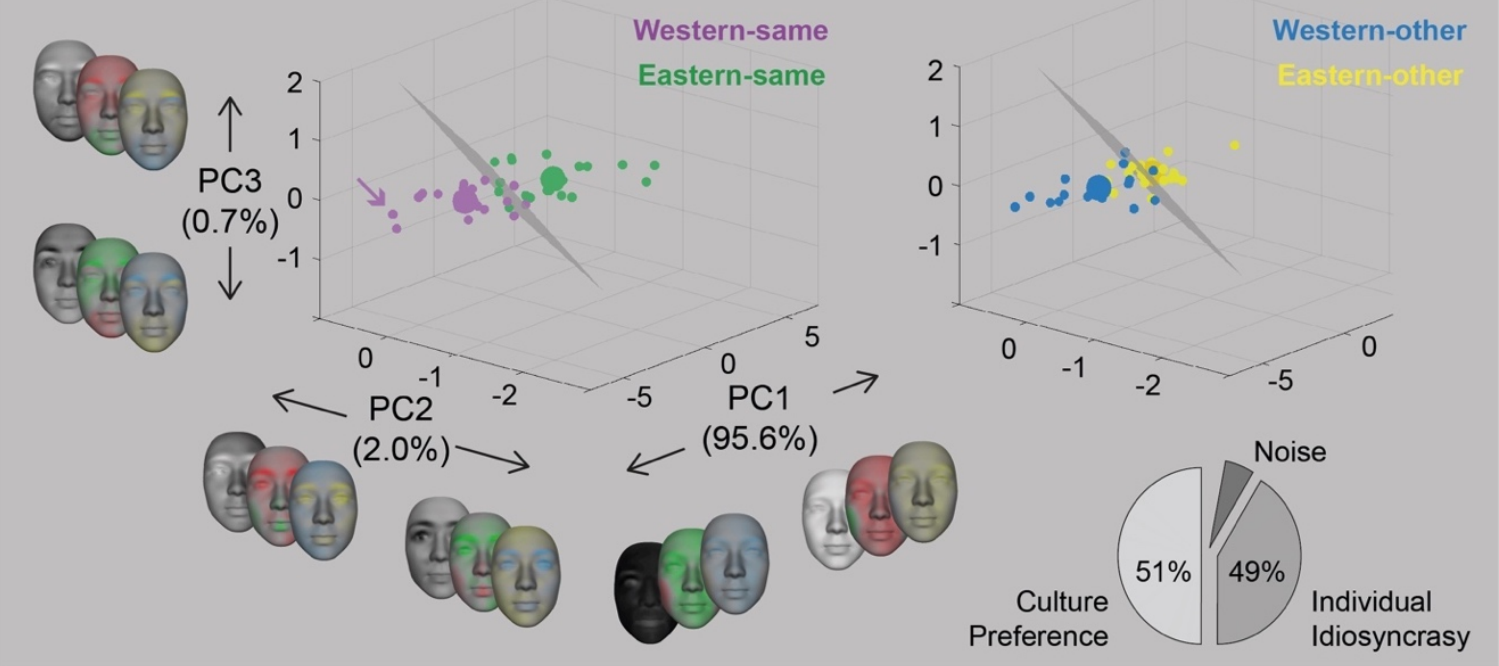

Figure 3. Cultural commonalities, preferences and individual differences.

A. Shape attractiveness. In the representational space, the three axes represent the first three multivariate shape (i.e. principal components, PC) that capture most $(74 \%)$ of the variance of individual participant models of attractive face shape. Left panel (own ethnicity models). The smaller colored dots represent 40 individual models that WE (in purple) and EA (in green) rated the faces of their own ethnicity. Large colored dots represent the group average of the models (c.f. Figure 1A \& B, panels 1-3). The grey surface illustrates the boundary (a linear SVM classifier) to separate the 20 Western-same vs. 20 Eastern-same shape models in this 3dimensional space, due to the cultural specificity of attractive shape features. Right panel (other ethnicity models). Same as left panel, using the same grey separation surface for reference, where blue (vs. yellow) dots denote each of individual models that WE (vs. EA) rated faces of another ethnicity. Pie chart shows $43 \%$ of model variance can be explained by 4 group averages (i.e. culture preference), with another $43 \%$ of model variance attributable to individuals' idiosyncratic preference. $B$ for $L^{*} a^{*} b^{*}$ complexion attractiveness, displayed in a same format as $A$. The grey surface illustrates the classification boundary between 20 Western-same vs. 20 Eastern-same complexion models in this 3-dimensional space. 
Cultural commonalities and differences. We compared cultures using the individual models of the same-ethnicity conditions (i.e. see left panel in Figure $3 A$ and $B$, where Western-same models are represented as the cloud of small purple dots and Eastern-same as a different cloud of small green dots). Nearly all models show the common preference for a smaller face and prominent forehead (represented by negative values for shape PC1 in both clouds). However, the models also show clear cultural differences as represented by the separation of the purple and green dots - Westerners show a preference for a pouty mouth and small nose (represented by shape PC3), darker skin and redder cheeks (represented by complexion PC1) whereas Easterners show preference for a smaller mouth and higher nose bridge (represented by shape PC3), a narrower face and pointy chin (represented by shape PC1) and lighter and yellower skin (represented by complexion PC1).

Individual differences. We also sought to characterize the idiosyncratic preferences of individual participants, quantify this variance and compare it to that explained by the group averages (see Materials and Methods, Analyses, Decomposing preference variance). For both shape and complexion, the group models (the large colored dots) and individual models (the small colored dots) explained a similar amount of variance (see pie charts in Figure $3 \mathrm{~A}$ and $3 \mathrm{~B}$ ). This decomposition, which shows the proportion of cultural vs. individual preferences within the representation space, demonstrates that a large proportion of individual preferences maintain essential variations of attractive features within a culture. These results align with existing studies using only variations of rating scores to full faces (10)(11).

Are cultural idiosyncrasies pervasive, transferring across other face ethnicities? Within the representational space, we further ascertained whether participants use the same cultural shape and complexion preferences when judging faces from another ethnicity (i.e. a transfer of their own-ethnicity preferences) or whether participant culture and face ethnicity interact, resulting in novel attractiveness features (other-ethnicity interactive preferences). We investigated the use own-ethnicity vs other-ethnicity features using the group models (see Materials and Methods, Analyses, Transferred and interactive preference). Figure 4 shows the results for each culture separately. As shown in Figure 4A-1 (left panel), Western shape preferences for other ethnicity faces (blue) lies at an intermediate location in the representation space between Western-same preferences (purple) and Eastern-same models (green). This indicates both a transfer of the Western-same own-ethnicity preferences for attractive face shapes across to the other ethnicity (e.g. a smaller nose), together with the development of new preferences as the WE culture interact with EA faces (e.g. a pointier chin). Figure 4A-2 illustrates the respective contributions of both transferred and interactive preferences to the Westernother model. A similar analysis applied to complexion in Figure 4A-1 (right panel) shows that the blue Western-other and the purple Western-same models overlap, indicating the full transfer of the WE complexion preferences (e.g. darker skin and redder cheeks) to EA faces. Likewise, the Eastern-other group model in yellow is located between the purple Western-same and green Eastern-same models, for both shape and complexion (see Figure 4B-1). Figure 4B-2 illustrates that Eastern-other transferred preferences for a higher nose bridge and pointy chin while reducing preference for lighter skin complexion.

In sum, cultural participants systematically shifted their preferences in the representation space, and in specific directions, with faces of different ethnicities. These effects 
illustrate both a transfer of cultural preferences for attractive face features to faces of another ethnicity, together with the development of new preferences that result from the interaction of culture and ethnicity.

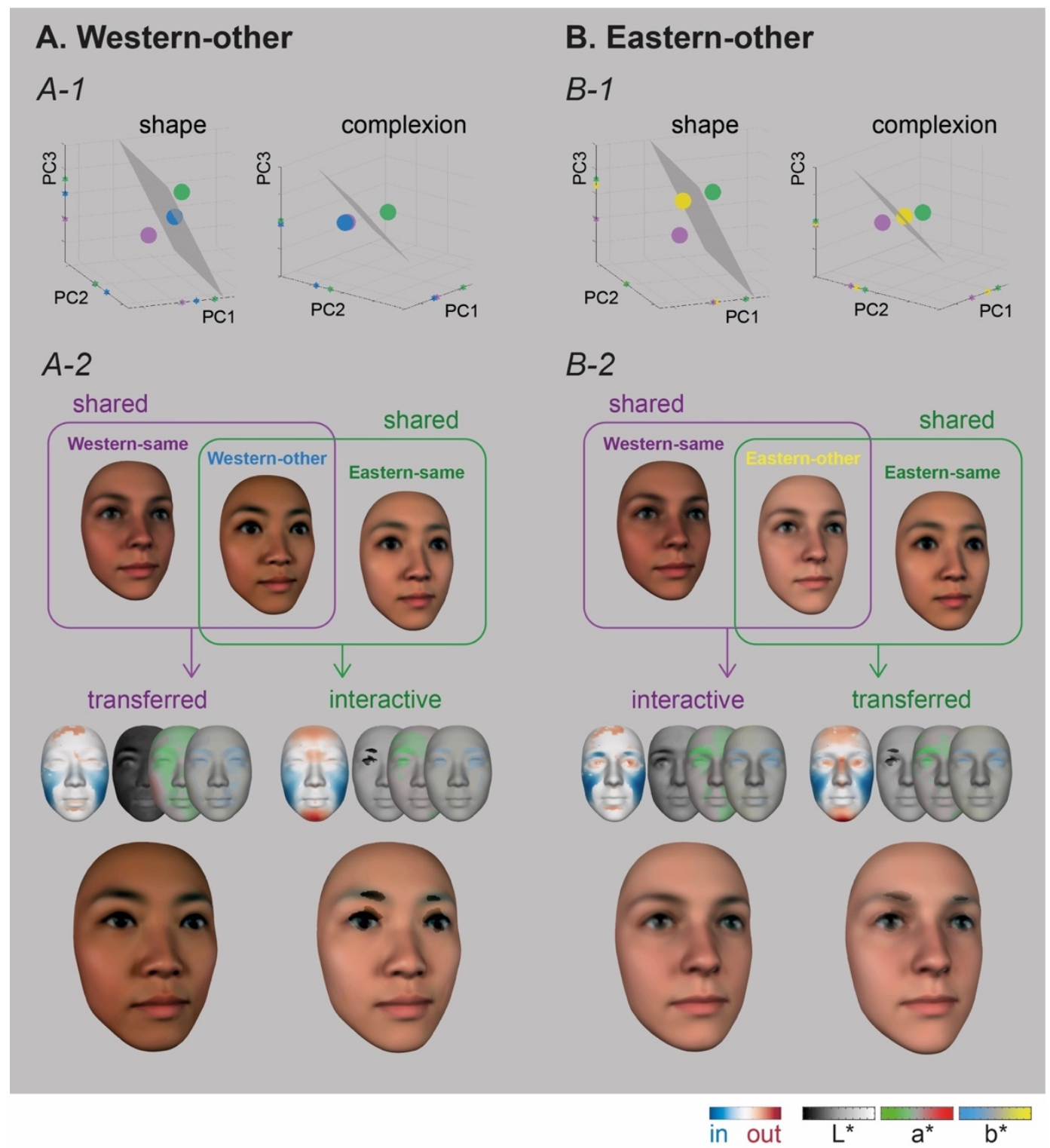

Figure 4. Transferred vs. interactive attractiveness features for other-ethnicity faces.

A. Western-other. $A-1$. In the representation space (shape, left panel; complexion, right panel) the large blue dot represents the group average of Western-other, in relation to the group average of Western-same (large purple dot) and Eastern-same (large green dot). Colored markers on each axis indicates the coordinate values on each model. $A-2$. Shared features between Westernsame and Western-other models in the purple set and indicate the transfer of attractive preferences from Western-same. Relatedly, shared features between Eastern-same and Western-other in the green set indicate the interactive preferences of WE males rating EA female faces. Smaller faces detail the transferred and interactive shape and complexion features. Larger faces at bottom render the transferred and interactive features. B. Eastern-other, color-coded in yellow and displayed in a same format as A. Deviations are displayed with a scale normalized to the maximum across all four conditions. 


\section{Discussion}

A longstanding debate is whether facial attractiveness is primarily based on universal and objective face properties (e.g. the face average or sexual dimorphism) or driven by idiosyncratic subjectivities at the level of a culture or an individual. We addressed the debate by modelling the face features that individuals within each of two cultures subjectively perceive as attractive when viewing same and other ethnicity faces, using a data-driven methodology that samples the natural distribution of face shape and complexion. Analysis of the resulting face models enabled us to address several key questions in this debate. First, we showed that the face average and sexual dimorphism do not explain facial attractiveness. Rather, attractive face features reside in their own space that explains cultural commonalities and individual idiosyncrasies. Second, we showed a common preference across cultures and face ethnicities for smaller female face shapes, with a complexion comprising high contrasted eye lines and eyebrows. We also showed that with same ethnicity faces, Westerners prefer a poutier mouth and a darker skin, whereas Easterners prefer a higher nose, pointier chin and lighter skin complexion. Analysis of other-ethnicity face models showed that these cultural preferences transferred to other ethnicity faces, together with the development of interactive preferences. Finally, we showed that individual participant idiosyncrasies in facial attractiveness preferences reflects a clear diversity that distributes in the space of attractive features. Thus, our results show that the features subtending facial attractiveness are away from the face average, exist in a feature space of their own, and reflect both cultural and individual diversity.

\section{D generative models of faces for control and synthesis}

We used the GMF to model the face features of attractiveness, which belongs to the broad class of 3D morphable, active appearance models (AAMs) of facial synthesis (29). AAMs represent the $3 \mathrm{D}$ surface and $2 \mathrm{D}$ complexion of faces as independent dimensions to afford better stimulus control than 2D, image-based face spaces, which are typically used to study facial attractiveness. Though other AAMs have been used to model facial attractiveness (e.g. (6)(30)), the GMF affords a tighter control with the categorical factors of face age, sex, ethnicity, and identity, whose face shape and complexion variances are explained away. Without such control, ambient and categorical factors of facial appearance would remain entangled. With the GMF, we ensured that all participants experienced a similar random distribution of random shape and texture residuals in each condition of our culture $\times$ face ethnicity experimental design. We derived models of facial attractiveness in a generative feature space. Thus, we can now transfer them to socially interactive digital agents in artificial intelligence, to bestow them with the ability to generate culturally suitable signs of face attractiveness with high utility in practical applications (see (31)(32) for a similar approach applied to facial expressions of emotion).

\section{Face attractiveness away from the average is an elaborate signal}

The challenge of modelling attractive face features is that humans have a distribution of subjective preferences for which there is no ground truth. Theories have therefore played a major role in proposing and testing specific hypotheses about the nature of human preferences (see (33)(34)(35)(36) for review). The main hypothesis is that attractive features are functional for mate choice, which in turn puts face shape and complexion under evolutionary pressures. One of the proposed choice is based on 
averages and a face is attractive because its features would be close to a population average and thereby linked to developmental stability and genetic diversity (37)(38). The averageness account has been massively investigated using 2D face photos and the composite of many faces. However, even if average faces are attractive does not mean that the most attractive faces are closer to the average. Also, the $2 \mathrm{D}$ photo averaging introduces blurring effect around shape edges, which loses the sensitivity to capture the 3D depth information. Another proposed choice is based on sexual dimorphism which instead argues that exaggerated secondary sexual characteristics are attractive because they advertise the quality of fertility and health (26)(27)(28). Again, many studies have been performed using 2D face images in front view and researchers correlated participant's attractiveness perception with the size of some manually selected local features (e.g. mouth width and height, face width at cheekbone).

Instead of testing a specific hypothesis, we used a data-driven approach to identify the space of face shape and complexion features that explain attractiveness. We aim to model the face attractiveness in the generative 3D space of faces (not the in 2D images). We found that this space differed from average face features and those representing sexual dimorphism. This is consistent with other studies arguing that the face average and sexual dimorphism do not capture attractiveness (6)(8). Preferences for attractive faces might have co-evolved with elaborate signalling in human communication (39). Our results show that attractive features are located at the outskirts of the natural distribution of face variations. Therefore, a choice bias for exaggerated shapes and complexions suggests an evolutionary drift (cf. peak drift, (20)(21)(40)). Such exaggerated forms could signal a higher level of health, such as a general preference for redder skin complexion in WE, which signals increased health with better oxygenated blood (41), super-stimulation (e.g. redder lips), and a preference for increased skin yellowness in EA, which potentially relates to a healthier diet with higher intake of carotenoids (41).

\section{Face attractiveness and cultural influences}

Our models highlight the influence of environmental and social factors on the perception of facial attractiveness. We found that different cultural norms exist for WE and EA, and these norms interacted with face ethnicity. We also showed individual variations within the cultural norms. Individual variations can arise from lifelong visual experience, as generally demonstrated with shape in visual perception (42)(43) (see (44) specifically for facial attractiveness). Here, the experience would be confined to the rarer outskirts of a face shape and complexion distributions. However, in the digital age, social media can promote such statistical learning in WE culture, with cultural stereotypes of a female pouty mouth and in EA culture, with a high nose bridge. Social stereotyping creates specific directional pressures on preference choice within populations, which in turn could support variations within and between cultures.

\section{Conclusion}

We modelled the perception of face attractiveness between and within cultures (as well as how attractiveness interacts with ethnicity). Our results directly inform and impact fundamental signalling theories of facial attractiveness in human psychology and ethology, by providing the actual representational contents of each individual and their 
diversity. Our approach opens the avenue to understand the nature of facial attractiveness and other subjective perceptions in the culturally diverse and highly complex social world.

\section{Materials and Methods}

Participants. We recruited a total of 80 male participants (40 White Western European, $\mathrm{WE}$, and 40 Chinese East Asian, EA, mean age $=23$, median age $=23, \mathrm{SD}=2.93$ ). $\mathrm{A}$ questionnaire assessed that all Westerners had minimal experience of non-Western cultures and all East Asians had resided in the UK for $<6$ months, with limited prior exposure to non-Eastern cultures (see Supplementary Information, Questionnaire). All participants had normal or corrected to normal vision, with no self-reported history or symptoms of synaesthesia, and/or any psychological, psychiatric or neurological condition that can affect face processing (e.g., depression, Autism Spectrum Disorder or prosopagnosia). Participants gave written informed consent prior to testing and received $£ 6$ per hour for their participation. The University of Glasgow College of Science and Engineering Ethics Committee provided ethical approval.

3D face stimuli. We used our Generative Model of 3D Faces (GMF, (19)) to synthesize 3,900 random 25-year old female faces equally split between WE and EA ethnicities. The GMF decomposes a 3D face (parametrized with 4,735 3D vertex coordinates for shape and $800 * 600$ RGB pixels for complexion, see Supplementary Fig. S1A) into two components: a categorical average defined by face age (i.e. set to 25 years old), ethnicity (WE vs. EA) and sex (set to female), plus a residual component that identifies each generated face. Two linear transformations underlie the generative model: (1) the extraction of a categorical average 3D face (represented by $4,735 \times 3$ shape coordinates and $800 \times 600 \times 3$ complexion pixels) that shares the features of 25 -year-old WE or EA females and (2) a Principal Components Analysis (PCA) of the multi-dimensional residuals (as 4,735x3 shape vertices and $800 \times 600 \times 3$ complexion pixels) that represent identity-specific features as a $467 \times 1$ shape vector coefficients (one per principal component) and a $467 \times 5$ matrix of complexion coefficients across 5 spatial frequency (SF) bands (again, one coefficient per principal component). To generate each face, we generated random identity residuals (separately for shape and complexion), by multiplying the generative PCs with random coefficients and added the categorical average of each ethnicity (i.e. WE or EA, see Supplementary Fig. S1B). At this stage, it is critical to understand that we added same 1,950 random identity residuals to the WE and EA female categorical averages, so that WE and EA stimuli share the same age, sex and random identity variations and only differed in their average ethnic information.

Procedure. Each trial started with a central fixation cross displayed for $1 \mathrm{~s}$, followed by a face presented on a black screen subtending an average of $9.5^{\circ} \times 6.4^{\circ}$ of visual angle, until response. We instructed participants to quickly rate the attractiveness of the face, based on their first impression, with a mouse click and using a 9-point rating scale displayed under the face (1, not attractive at all; 9, very attractive). Following response and a 500 ms blank interval a new trial would begin. The experiment comprised 1,950 trials in a $2 \times 2$ between-participants design, so that each cultural participant (EA or WE) would rate faces only from one ethnicity (i.e. either WE or EA faces). Across trials, the $1,9503 \mathrm{D}$ stimuli appeared on the screen presented in one of three evenly distributed 
viewpoints (-30, 0 and $+30^{\circ}$ of rotation in depth). The experiment comprised a total of 39 randomly allocated blocks of 50 trials that each participant performed over 2-3 days. Participants sat in a dimly lit room and used a chin rest to maintain a fixed viewing distance. We used the Psychtoolbox (45)(46) for MATLAB R2018a to control the experiment.

\section{Analyses.}

Linear regression model. We performed linear regression analyses independently for each participant. An experimental trial paired the trial-specific stimulus parameters (a 467-dimensional vector of random shape coefficients; a $467 \times 5$ dimensional matrix of random complexion coefficients) with the corresponding participant's attractiveness rating response (a value between 1 and 9). Across trials, we linearly regressed the stimulus parameters with the participant's ratings, separately for each shape and complexion dimension (RobustFit, Matlab 2018a) as in Equation (1) below.

$$
P C_{j} \text { coefficient }=\beta 1+\beta 2 \times \text { Ratings }(1)
$$

Equation (1) delivered a linear model with $\beta 1$ and $\beta 2$ coefficients for each dimension of 3D shape and 2D complexion that generated the face stimuli. These $\beta 1$ and $\beta 2$ coefficients therefore model and explain how variations of face shape and complexion linearly relate to variations of face attractiveness perception in each participant. We called these $\beta 1 \mathrm{~s}$ and $\beta 2 \mathrm{~s}$ the participant's model of face attractiveness. This model is multivariate, comprising 467 shape dimensions and $467 \times 5$ complexion dimensions, separately for the $\beta 1$ and $\beta 2$ coefficients that multiply the principal components of shape and complexion.

We repeated these regression analyses at the finer granularity of individual vertices and pixels, to address with univariate analyses effects that the multivariate analyses might hide. For each shape vertex $(N=4,735)$, we linearly and independently regressed its $X, Y$ and $Z 3 D$ face coordinates on the corresponding attractiveness ratings; likewise, for each of complexion pixel ( $N=480,000$ downsampled to 30,000 pixels), we linearly and independently regressed its $L, a^{*}, b^{*}$ channels on the corresponding attractiveness ratings $(p<0.05$, two-tailed, for the $\beta 2 s$, corrected for multiple comparisons with the false discovery rate method, across all $4735^{\star} 3$ vertex coordinates and $200^{*} 150^{*} 3 \mathrm{~L}^{*} \mathrm{a}^{*} \mathrm{~b}^{*}$ pixel values). Supplementary Fig. S2 reports these individual models, showing that a high correspondence between the multivariate and the univariate linear regressions.

Reconstructing attractive and unattractive faces. In the linear regression model of Equation 1, $\beta 2$ coefficients control perceived face attractiveness at the level of individual participants. Remember that our experiment comprised 4 conditions (Western-same and other; Eastern- and other). For each condition, we computed a group-level model by averaging the $\beta 1$ and $\beta 2$ coefficients (of the multivariate linear regressions) of each individual participant. We then rendered positively (for attractive) and negatively (for unattractive) amplified shape and complexion $\beta 2$ s of each group average (see Panel 7 and 9 in Figure 1).

Face average and feature distribution. The GMF represents each rated stimulus as a categorical average plus an identity residual component, separately for shape and 
complexion (see Materials and Methods, 3D face stimuli). Here, in four steps we addressed the question of the location of attractive features in the distribution of shape and complexion variations, separately for each of our 4 conditions.

Step 1: We selected for each participant the stimuli rated as highly attractive, and pooled these across all 20 participants of the considered experimental condition to form the attractive stimuli set.

Step 2: We extracted, per shape and complexion PC, the identity coefficient that approximates the modal value of all its identity coefficients in the attractive stimuli from Step 1. We then used the modal coefficients (i.e. $467 \times 1$ for shape and $467 \times 5$ for complexion identity residuals) plus its categorical average to synthesize a face that describe the attractive features (i.e. 4763 shape vertices and 800x600 complexion pixels' $L^{*} a^{*} b^{*}$ ) of these attractive stimuli.

Step 3: We computed the mean and standard deviation of each face vertex and complexion across the 1,950 experimental stimuli.

Step 4: We z-scored attractive features per vertex and pixel $L^{*} a^{*} b^{*}$ that computed in Step 2, using their mean and standard deviations computed in Step 3.

Sexual dimorphism and attractiveness. In the group model, the $467 \beta 2$ s of shape and $467 \times 5 \beta 2$ s of complexion specify multivariate directions of shape and complexion change (in the GMF PC space) that characterize an attractive female face away from the categorical average. To examine the relationship between this direction of change (for shape and complexion) and that of femininity (i.e. sexual dimorphism) we proceeded as follows. First, we computed the direction of sexual dimorphism as the per-vertex (for shape) and per pixel (for complexion) difference between average female and average male faces, independently for WE and EA faces. Second, we projected this male-female difference into the GMF PC space to reparametrize sexual dimorphism as a $467^{*} 1$ shape vector and a $457 \times 5$ complexion matrix. Next, now that both attractiveness and sexual dimorphism were two directions in the same space, we computed their similarity with vector cosine. Specifically, we reduced the dimensionality of the vector cosine computation (using the elbow method, see Supplementary Information) to keep only the eigenvectors with significant eigenvalues-i.e. for shape, 42 dimensions; for complexion 116 dimensions split as follows: 10 dimensions for SF1; 18 for SF2; 30 for SF3; 41 for SF4; 17 for SF5. We derived a vector (42-dimensional for shape; 116-dimensional for complexion) that specifies the changes in attractiveness $(\vec{A})$ not explained by the changes in sexual dimorphism $(\vec{D})$. This vector is the residual attractiveness vector $(\vec{A})$, following its projection on the sexual dimorphism vector $(\vec{D})$, mathematically defined as equation (2):

where $\widehat{D}$ is the normalized vector of $\vec{D}$.

$$
\vec{A}_{\text {resi }}=\vec{A}-(\vec{A} \cdot \widehat{D}) \cdot \widehat{D}
$$

Components of attractiveness. To characterize the representation space, we applied PCA to the 80 individual models, separately for shape and complexion, vectorizing the models at vertex- and pixel-level granularities-i.e. 14,205-dimensional shape vectors, from 4,735 vertices * 3 coordinates and 140,000-dimensional complexion vectors, from $800^{*} 600$ pixels * 3 dimensions of $L^{*} a^{*} b^{*}$. Supplementary Fig. S6 show the significant shape $(\mathrm{N}=8$, explained $94.23 \%$ of total variance) and complexion PCs $(\mathrm{N}=$ 3 , explained $98.32 \%$ of total variance) PCs determined by the elbow method. We plotted 
only the first three shape PCs in Figure 3 for illustration purpose. We used all these significant PCs as the face space of attractiveness for subsequent analyses.

Decomposing preference variance. Using the representation space, we projected each high-dimensional model into 8 shape PC dimensions and 3 complexion PC dimensions, using the resulting scores for further analyses. We decomposed the variance of these component scores across all models into the variance explained: 1) by the mean of their experimental condition (i.e. the cultural preference), and 2) by each individual model (i.e. the individual preference).

To do this, we first employed bootstrap resampling to quantify the sampling variance of the individual models obtained with our experimental procedure. We calculated 20 bootstrap models for each participant (c.f. Materials and Method, Analyses, Linear regression model) sampling with replacement 1,950 trials. Then, we repeated the PCA analysis as in Materials and Method, Analyses, Components of attractiveness, this time using 1,600 models (i.e. 80 participants $\times 20$ bootstrapped models $=1600)$. Supplementary Fig. $S 7$ reports the feature space of face attractiveness of 1,600 models. Next, we decompose the variance of each shape and complexion component:

Step 1: We calculated the total variance $S S_{\text {total }}$, using the sum of squared distance from the overall mean $(S S)$

Step 2: We removed the corresponding group mean of the component, and calculated the sum of squared residuals $S S_{\text {resi1 }}$ not explained by the 4 group means. The proportion of variance (i.e. the $R^{2}$ ) explained by group means is given by:

$$
R_{\text {group }}^{2}=1-S S_{\text {resi1 }} / S S_{\text {total }}
$$

Step 3: We then removed, the participant-level residual mean, and calculated the $S S$ of further left residuals $S S_{\text {resi2 }}$ not explained by 80 participants' means. The proportion of variance explained by individual model thus equals to (4):

$$
R_{\text {participant }}^{2}==S S_{\text {resi1 }} / S S_{\text {total }} \times\left(1-S S_{\text {resi } 2} / S S_{\text {resi1 } 1}\right)
$$

Across 8 shape components (and 3 complexion components, separately), we calculated the weighted sum of $R^{2}$ group and $R^{2}$ participant scaled by the variance explained by each PC, and obtained overall in the attractiveness PC space the proportion of culture preference vs. individual preference (see pie chart in Figure 3). Note that the remaining unexplained variance is related to the sampling variance of our individual models, revealed by the bootstrap resampling.

To determine the threshold of $R^{2}$ group for each attractiveness dimension, in each of 200 iterations, we shuffled 1600 models across 4 conditions while keeping in each participant their bootstrapped models, and repeated Step 2 above. The $200 R^{2}$ outcomes capture the null distribution that there is no group difference. We computed 99.375 percentile of the null distribution as the threshold for shape $R^{2}$ group, as we corrected the multiple comparison for 8 shape PCs (one-tailed, $p<0.05$, Bonferroni corrected--i.e. $99.375=100-5 / 8$ ). Likewise, we computed the 98.34 percentile of 200 $R^{2}$ outcomes for complexion $R^{2}$ group - -i.e. $98.34=100-5 / 3$. Across dimensions (8 for shape and 3 for complexion), we computed the weighted sum of the thresholds to obtain the overall threshold to determine significance (see Supplementary Fig. S8.

To calculate the threshold of $R^{2}{ }_{s u b j}$, we kept the 4 conditions in their right place, but shuffled all 400 models in each condition. We repeated Step3 in each of 200 
iterations and calculated the threshold in the same way as we did for $R^{2}$ group (see Supplementary Fig. S8).

Transferred and interactive preference. To separate the respective contributions of transferred and interactive features in Western-other and Eastern-other models, we proceeded as follows. First, we computed the group models of each experimental condition by averaging the $\beta 2$ coefficients across the 20 participants in vertex and pixel $L^{*} a^{*} b^{*}$ spaces. For each vertex of (and pixel $L^{*} a^{*} b^{*}$ ) of the Western-other (and Easternother) group model we computed whether it had the same sign as the Western-same model and, independently, as the Eastern-same model. A transferred feature of Western-other (and Eastern-other) would share $\beta 2$ signs with Western-same (vs. Eastern-same). An interactive feature of Western-other (vs. Eastern-other) would share $\beta 2$ signs with Eastern-same (vs. Western-same).

Model Validation. We validated each individual model, using their shape and complexion projection in the representation space of attractiveness, to predict the pertrial attractiveness judgements of individual participants. We did so to test that our representation space better predicts participant's ratings than the face average and the sexual dimorphism hypothesis.

For each participant, we segmented the full trials $(N=1,950)$ into 13 adjacent blocks and performed a 13-folds blockwise cross validation. In each of the 13 iterations, we proceeded in 4 steps, separately for shape and complexion:

Step 1: We built a linear regression model using trials in 12 adjacent blocks (i.e. training set, $\mathrm{N}=1,800$ trials), as in Materials and Methods, Analyses, Linear regression model.

Step 2: We used the model from Step1 and another 79 models of left out participants (from above Materials and Methods, Analyses, Linear regression model) to derive the space of attractiveness (same as Materials and Methods, Analyses, Components of attractiveness).

Step 3: For each stimulus, we extracted its identity residual by removing the GMF categorical average and reparametrized the residuals into the attractiveness space (as an 3-dimensional shape vector and a 8-dimensional complexion vector) and used these as the predictor $X$. We defined $Y$ as the attractiveness rating to be predicted based on $X$ (separately for shape and complexion).

Step 4: We trained a Generalized Linear Model (GLM) to predict the attractiveness ratings $Y$ from the predictors $X$-i.e. $Y^{\prime}=\exp (X b)$. We used the log link function with a Gamma error distribution to account for the non-negative and skewed ratings (only few trials were rated as highly attractive). We fitted the GLM model using the method of iterative reweighted least squares implemented in fitglm in Matlab 2019a. We tested the model prediction performance using the trials in the leave-out block $(\mathrm{N}=$ 150 ) and checked the ranking consistency (Kendall $\tau$ rank correlation) between each participant's actual ratings and the GLM predicted ratings. For comparison, we also trained 2 alternative GLM models:

Alternative model 1 (averageness): the predictor $X$ for each stimulus was the global distance of its shape (or complexion) to the GMF face average of its experimental condition, where we computed shape global distance as the Euclidean distance between the stimulus position to the center of the space (i.e. the GMF average face, a distance between two 467-dimensional shape coefficients). We repeated this distance 
computation for complexion in the vectorized 2,335-dimension GMF complexion space $\left(2,335=467^{\star} 5\right)$.

Alternative model 2 (sexual dimorphism): the predictor $X$ for each stimulus was the scalar projection of its GMF shape PCs (or complexion PCs) onto the shape (or complexion) sexual dimorphism vector $(\vec{D})$ (see sexual dimorphism calculation as above). The vector scalar projection was computed using a reduced set of GMF PCs, i.e. for shape, 42 dimensions; for complexion 116 dimensions (10 dimensions for SF1; 18 for SF2; 30 for SF3; 41 for SF4; 17 for SF5), defined by the elbow method as we did above.

We also trained and tested the alternative models in a 13-folds block-wise crossvalidation. Supplementary Fig. S9 shows the prediction performance quantified by Kendall's $\tau$ rank correlation of all models, in which we showed our attractiveness models predict subjective ratings significantly better than average (pair-wised t test, $p<0.001$ for both shape and complexion, Bonferroni correction) and sexual dimorphism models (pairwised $t$ test, $p<0.001$ for both shape and complexion, Bonferroni correction).

\section{Acknowledgments}

P.G.S. received support from the Wellcome Trust (Senior Investigator Award, UK; 107802) and the Multidisciplinary University Research Initiative/Engineering and Physical Sciences Research Council (USA, UK; 172046-01). The funders had no role in study design, data collection and analysis, decision to publish or preparation of the manuscript.

\section{References}

1. K. Dion, E. Berscheids, E. Walster, What is Beautiful is Good. J. Pers. Soc. Psychol., 285290 (1972).

2. J. H. Langlois, L. A. Roggman, Attractive Faces Are Only Average. Psychol. Sci. 1, 115121 (1990).

3. K. Grammer, R. Thornhill, Human (Homo sapiens) facial attractiveness and sexual selection: The role of symmetry and averageness. J. Comp. Psychol. 108, 233-242 (1994).

4. D. I. Perrett, et al., Symmetry and Human Facial Attractiveness. Evol. Hum. Behav. 20, 295-307 (1999).

5. D. I. Perrett, et al., Effects of sexual dimorphism on facial attractiveness. Nature 394, 884887 (1998)

6. C. P. Said, A. Todorov, A Statistical Model of Facial Attractiveness. Psychol. Sci. 22, 1183-1190 (2011).

7. D. I. Perrett, K. A. May, S. Yoshikawa, Facial shape and judgements of female attractiveness. Nature 368, 239-242 (1994).

8. I. J. Holzleitner, et al., Comparing theory-driven and data-driven attractiveness models using images of real women's faces. J. Exp. Psychol. Hum. Percept. Perform. 45, 15891595 (2019).

9. I. M. Scott, et al., Human preferences for sexually dimorphic faces may be evolutionarily novel. Proc. Natl. Acad. Sci. 111, 14388-14393 (2014).

10. L. Germine, et al., Individual Aesthetic Preferences for Faces Are Shaped Mostly by Environments, Not Genes. Curr. Biol. 25, 2684-2689 (2015). 
11. J. Hönekopp, Once more: Is beauty in the eye of the beholder? Relative contributions of private and shared taste to judgments of facial attractiveness. J. Exp. Psychol. Hum. Percept. Perform. 32, 199-209 (2006).

12. T. Tanaka, J. Mikuni, D. Shimane, K. Nakamura, K. Watanabe, Accounting for Private Taste: Facial shape analysis of Attractiveness and Inter-individual Variance in 2020 12th International Conference on Knowledge and Smart Technology (KST), (2020), pp. 203206.

13. I. S. Penton-Voak, et al., Menstrual cycle alters face preference. Nature 399, 741-742 (1999).

14. I. S. Penton-Voak, D. I. Perrett, Female preference for male faces changes cyclically: Further evidence. Evol. Hum. Behav. 21, 39-48 (2000).

15. A. C. Little, B. C. Jones, D. M. Burt, D. I. Perrett, Preferences for symmetry in faces change across the menstrual cycle. Biol. Psychol. 76, 209-216 (2007).

16. A. C. Little, B. C. Jones, Variation in facial masculinity and symmetry preferences across the menstrual cycle is moderated by relationship context. Psychoneuroendocrinology 37, 999-1008 (2012).

17. B. C. Jones, et al., No Compelling Evidence that Preferences for Facial Masculinity Track Changes in Women's Hormonal Status. Psychol. Sci. 29, 996-1005 (2018).

18. B. C. Jones, A. C. Hahn, L. M. DeBruine, Ovulation, Sex Hormones, and Women's Mating Psychology. Trends Cogn. Sci. 23, 51-62 (2019).

19. J. Zhan, O. G. B. Garrod, N. van Rijsbergen, P. G. Schyns, Modelling face memory reveals task-generalizable representations. Nat. Hum. Behav. 3, 817-826 (2019).

20. A. Arak, M. Enquist, Hidden preferences and the evolution of signals. 7.

21. M. J. Ryan, M. E. Cummings, Perceptual Biases and Mate Choice. Annu. Rev. Ecol. Evol. Syst. 44, 437-459 (2013).

22. Y. Gao, J. Niddam, W. Noel, B. Hersant, J. P. Meningaud, Comparison of aesthetic facial criteria between Caucasian and East Asian female populations: An esthetic surgeon's perspective. Asian J. Surg. 41, 4-11 (2018).

23. S. Liew, et al., Consensus on Changing Trends, Attitudes, and Concepts of Asian Beauty. Aesthetic Plast. Surg. 40, 193-201 (2016).

24. Q. (Vivi) Xie, M. Zhang, White or tan? A cross-cultural analysis of skin beauty advertisements between China and the United States. Asian J. Commun. 23, 538-554 (2013).

25. E. P. H. Li, H. J. Min, R. W. Belk, J. Kimura, S. Bahl, Skin Lightening and Beauty in Four Asian Cultures. 7.

26. N. Barber, The evolutionary psychology of physical attractiveness: Sexual selection and human morphology. Ethol. Sociobiol. 16, 395-424 (1995).

27. M. j Law Smith, et al., Facial appearance is a cue to oestrogen levels in women. Proc. $R$. Soc. B Biol. Sci. 273, 135-140 (2006).

28. J. R. Wheatley, et al., Women's faces and voices are cues to reproductive potential in industrial and forager societies. Evol. Hum. Behav. 35, 264-271 (2014).

29. A. J. O'Toole, C. D. Castillo, C. J. Parde, M. Q. Hill, R. Chellappa, Face Space Representations in Deep Convolutional Neural Networks. Trends Cogn. Sci. 22, 794-809 (2018).

30. K. Nakamura, K. Watanabe, Data-driven mathematical model of East-Asian facial attractiveness: the relative contributions of shape and reflectance to attractiveness judgements. R. Soc. Open Sci. 6, 182189 (2019).

31. C. Chen, et al., Equipping social robots with culturally-sensitive facial expressions of emotion using data-driven methods in 2019 14th IEEE International Conference on Automatic Face Gesture Recognition (FG 2019), (2019), pp. 1-8.

32. C. Chen, et al., Reverse Engineering Psychologically Valid Facial Expressions of Emotion into Social Robots in 2018 13th IEEE International Conference on Automatic Face Gesture Recognition (FG 2018), (2018), pp. 448-452. 
33. R. Thornhill, S. W. Gangestad, Facial attractiveness. Trends Cogn. Sci. 3, 452-460 (1999).

34. G. Rhodes, The Evolutionary Psychology of Facial Beauty. Annu. Rev. Psychol. 57, 199226 (2006).

35. A. C. Little, B. C. Jones, L. M. DeBruine, Facial attractiveness: evolutionary based research. Philos. Trans. R. Soc. B Biol. Sci. 366, 1638-1659 (2011).

36. B. Fink, I. Penton-Voak, Evolutionary Psychology of Facial Attractiveness. Curr. Dir. Psychol. Sci. 11, 154-158 (2002).

37. R. Thornhill, S. W. Gangestad, Human facial beauty: Averageness, symmetry, and parasite resistance. Hum. Nat. 4, 237-269 (1993).

38. G. Rhodes, et al., Do facial averageness and symmetry signal health? Evol. Hum. Behav. 22, 31-46 (2001).

39. T. Guilford, M. S. Dawkins, Receiver psychology and the evolution of animal signals. Anim. Behav. 42, 1-14 (1991).

40. M. Enquist, A. Arak, Selection of exaggerated male traits by female aesthetic senses. Nature 361, 446-448 (1993).

41. I. D. Stephen, M. J. Law Smith, M. R. Stirrat, D. I. Perrett, Facial Skin Coloration Affects Perceived Health of Human Faces. Int. J. Primatol. 30, 845-857 (2009).

42. S. Panis, J. Wagemans, H. P. O. de Beeck, Dynamic Norm-based Encoding for Unfamiliar Shapes in Human Visual Cortex. J. Cogn. Neurosci. 23, 1829-1843 (2011).

43. , Norm-based face encoding by single neurons in the monkey inferotemporal cortex | Nature (July 27, 2020).

44. G. Rhodes, L. Jeffery, T. L. Watson, C. W. G. Clifford, K. Nakayama, Fitting the mind to the World: Face Adaptation and Attractiveness Aftereffects. Psychol. Sci. 14, 558-566 (2003).

45. D. H. Brainard, The Psychophysics Toolbox. Spat. Vis. 10, 433-436 (1997).

46. M. Kleiner, D. H. Brainard, D. G. Pelli, What's new in Psychtoolbox-3? Percept. 36 ECVP Abstr. Suppl. 


\section{Supplementary Information}

3D face database. The face database comprised 265 females, 202 males, 296 Western Caucasian, 164 East Asian, 7 Black African, age between 16 and 88, SD = 13, scanned in-house with a Di4D face capture system, at a high resolution in shape $(4,7353 \mathrm{D}$ vertex coordinates) and texture $(800 * 600$ RGB pixels, see Supplementary Fig. S1A). All 3D models were in full color with hair removed, posing with a neutral facial expression.

\section{Questionnaire}

Western European Participants. Each potential Western European participant answered the following questionnaire. We selected only those who answered 'no' to all questions, or who answered 'no' to question $2 \& 3$ and had been to non-Western* country for only a short vacation (i.e. less than 2 weeks).

1. How long you have spent in a non-Western* country IN TOTAL since you were 10 years old?

2. Have you ever been in close contact with any non-Western* person(s) who has been your friend or acquaintance for quite some time?

3. Have you ever been involved with any non-Western* culture societies/groups?

* by Western groups/countries, we are referring to Europe (Eastern and Western), USA, Canada, United Kingdom, Australia and New Zealand.

East Asian Participants. Each potential East Asian participant answered the following questionnaire. We selected those who entered UK less than 6 months (question 1) and answered 'no' to all questions.

1. At what date did you first enter the UK?

2. How long you have spent in a non-Eastern* country IN TOTAL since you were 10 years old?

3. Have you ever been in close contact with any non-Eastern* person(s) who has been your friend and acquaintance for quite some time?

4. Have you ever been involved with any non-Eastern* culture societies/groups?

* by Eastern groups/countries, we are referring to China, Japan, Korea, Thailand and Taiwan.

\section{Elbow Method}

\section{Sexual dimorphism and attractiveness}

We ranked the eigenvalues of GMF PCs and plotted the eigenvalue curve (see Supplementary Figure S10A, black curve). We determined the threshold point (see red point in Supplementary Figure S10A) on the curve that has the furthest distance (see d' in Supplementary Figure S10A) from the straight line connecting the first and last point of the eigenvalue curve (see dash line in Supplementary Figure S10A). Supplementary Figure S10B shows the feminine features represented by the significant components against the representations of the full components, demonstrating the reliability of this method.

\section{Components of attractiveness}

We ranked the eigenvalues of attractiveness PCs and determined the threshold point as we introduced above (c.f. Supplementary Figure S10A), separately for shape and complexion. Supplementary Figure S10C shows the attractive features represented by the significant components against the representations of the full components, demonstrating the reliability of this method. 


\section{A. 3D Face Parameters}

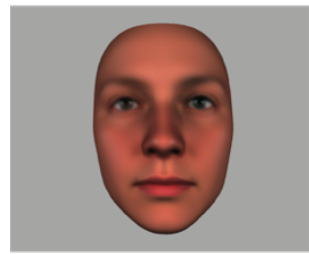

3D Face

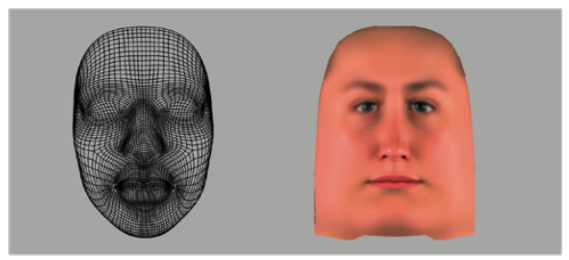

3D Shape

2D Complexion

\section{B. Random Face Generation}

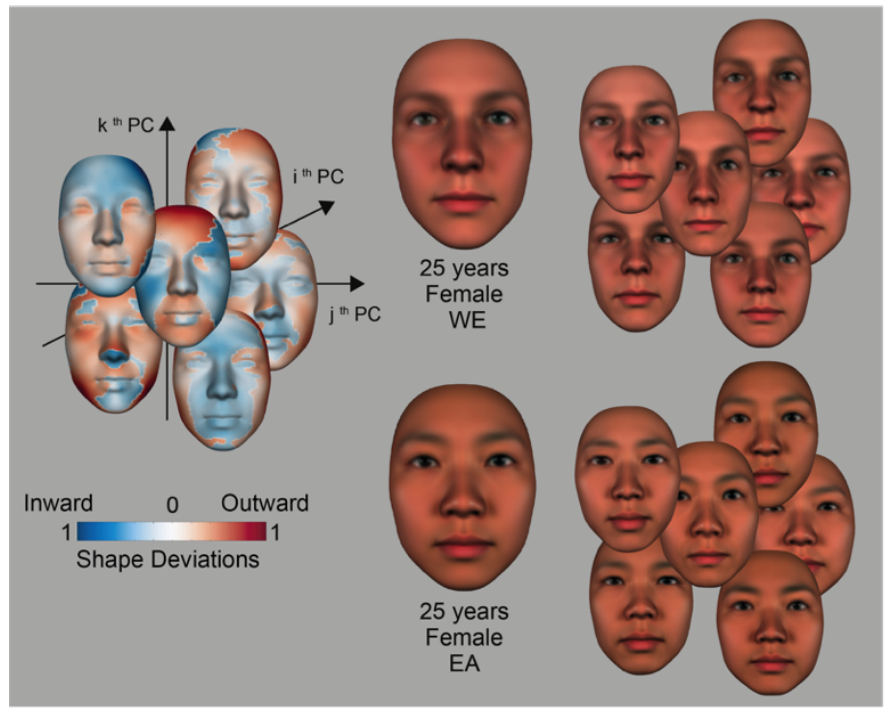

Random Identity Residuals + GLM (age,gender,ethnicity) = Random Identities

\section{Real Face Reconstruction}

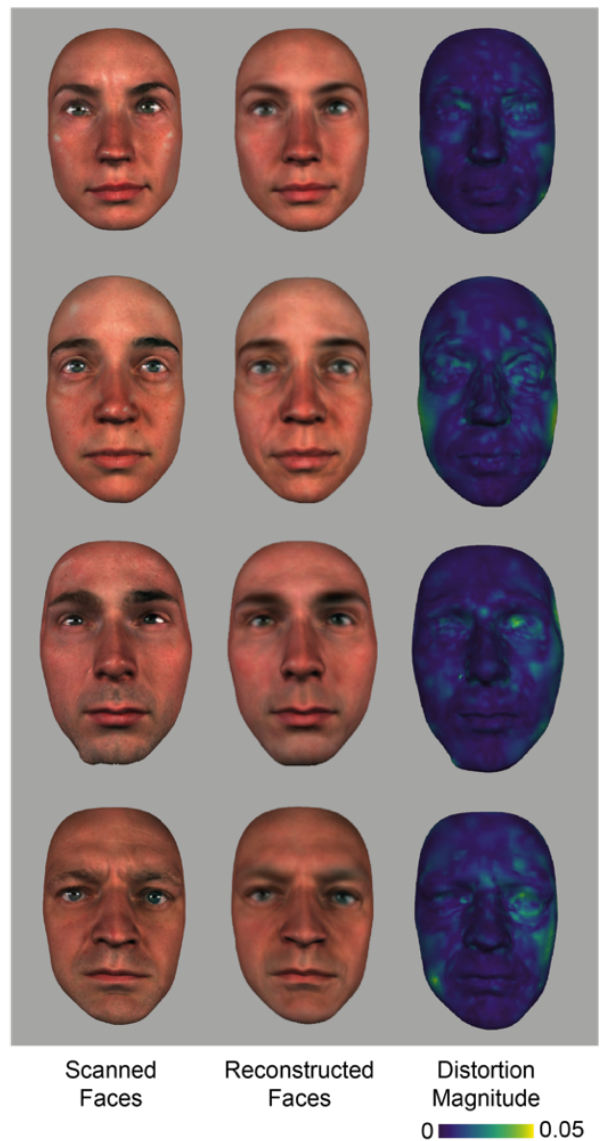

Fig. S1. A. 3D face parameters. We parametrized the shape of a 3D face with the 3D coordinates of 4,735 vertices and its complexion with $800^{*} 600^{\star} 3$ RGB 2D pixels. B. Random face generation. In the GMF, we synthesized each face stimulus as the sum of a categorical average capturing the factors of face sex (female), ethnicity (WE or EA), age (25 years old) and their interactions plus a random component of 3D shape identity (and 2D complexion component, not illustrated here). Across the experiment, all face stimuli shared the same categorical average and had a unique and randomly defined identity. The color scale indicates the magnitude of 3D shape deviations from the categorical average (normalized by the maximum deviation). C. Real face reconstruction. We scanned 4 new faces and fitted each within the GMF. The low distortion magnitudes indicate, vertex per vertex, the high quality of the reconstructed GMF fit to the ground truth scanned faces. 


\section{A. Individual Models}

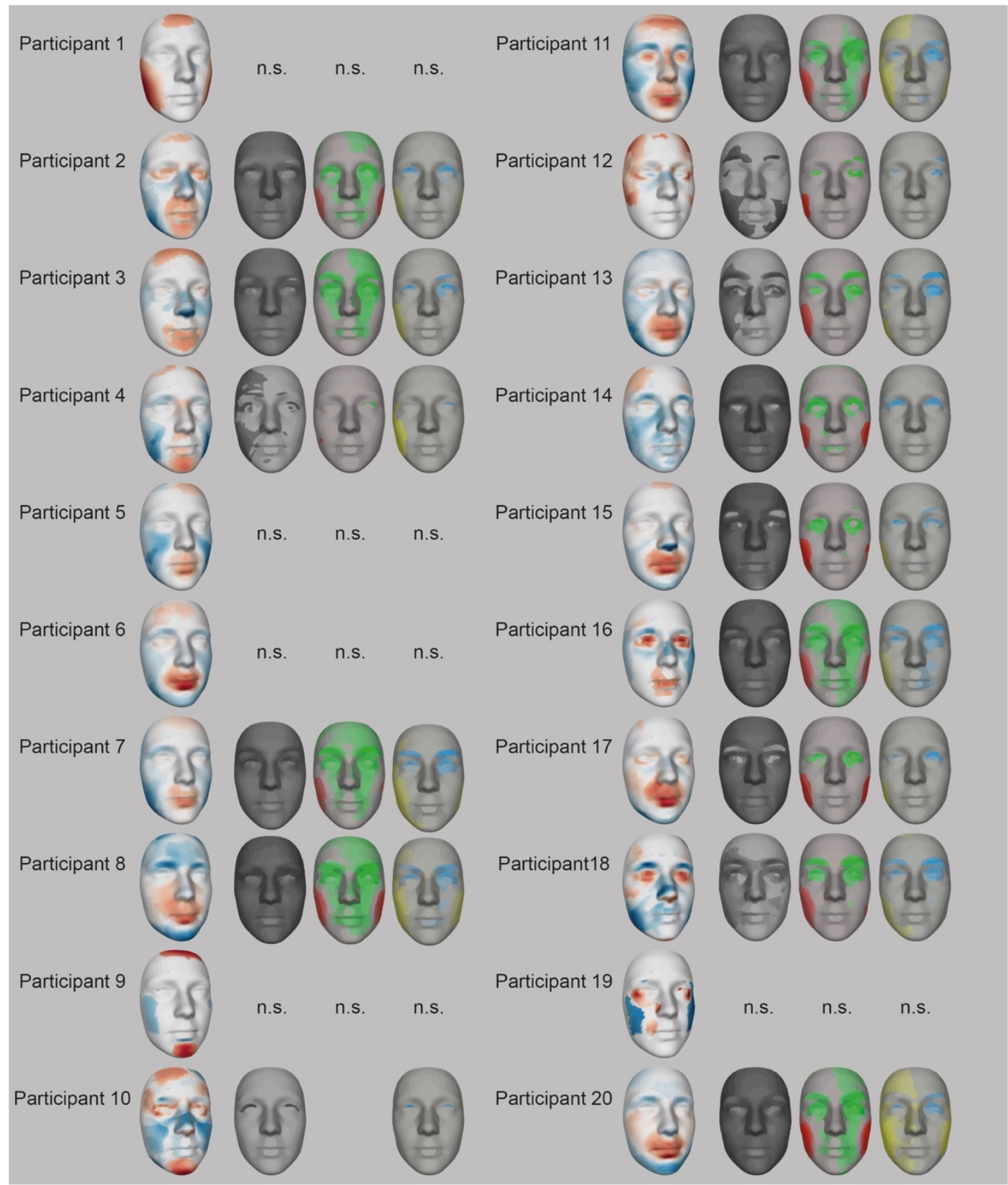

\section{B. Group Model}

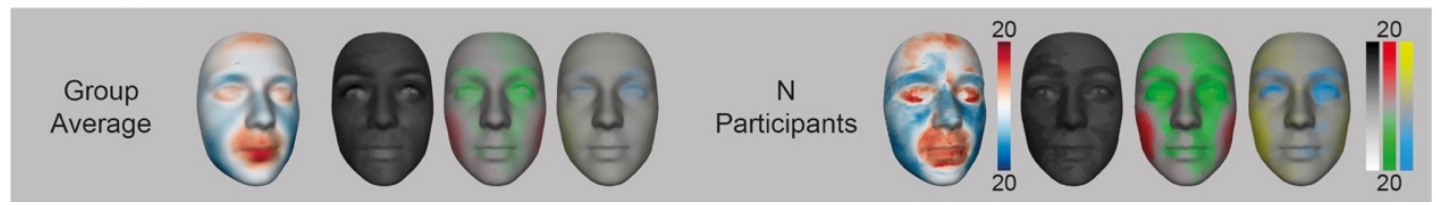

Fig. S2-1. A. Individual model of Western-same. For each participant, we colored significant shape vertices and pixels' $L^{*} A^{*} B$ according to their effect size (i.e. slope $\beta$ in the linear model). $B$. Group model of Western-same. Left. Averaged effect size across participants. Right. Number of participants, per vertex and pixel $L^{*} A^{*} B$, with significant slope $\beta s$. 


\section{A. Individual Models}

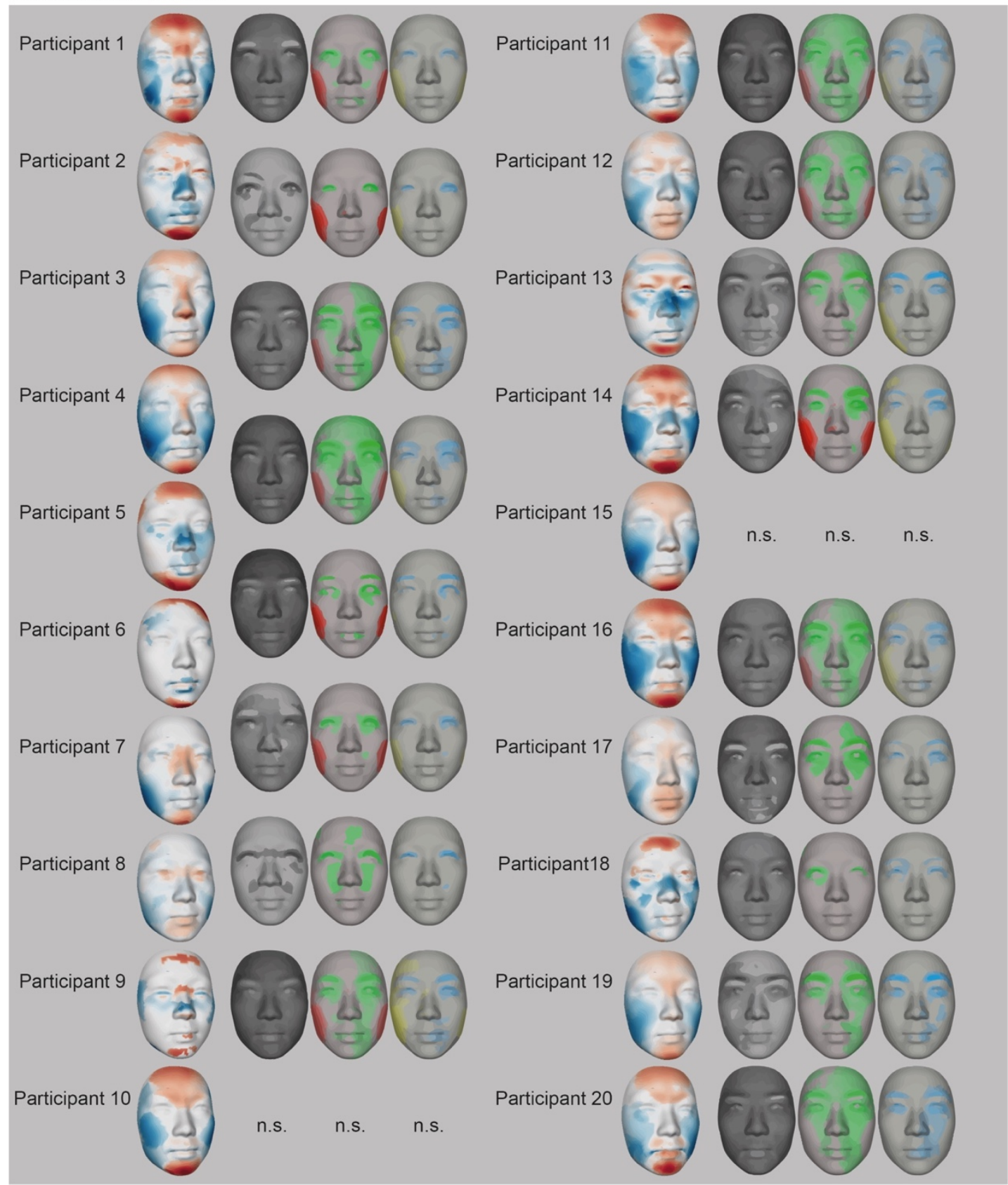

\section{B. Group Model}

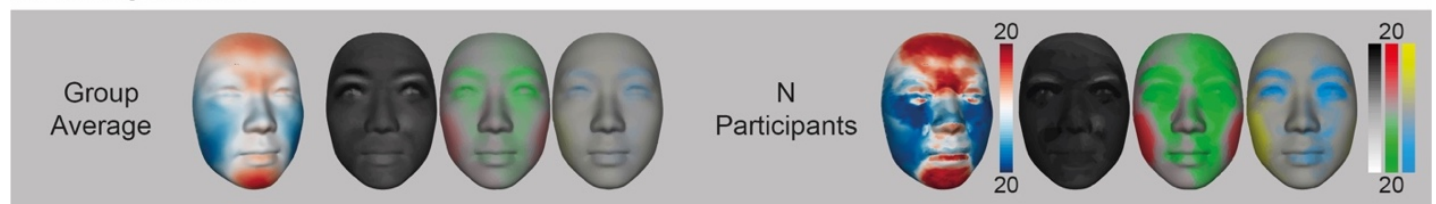

Fig. S2-2. A. Individual models of Western-other. B. Group model of Western-other. Same caption as Figure S2-1. 
A. Individual Model

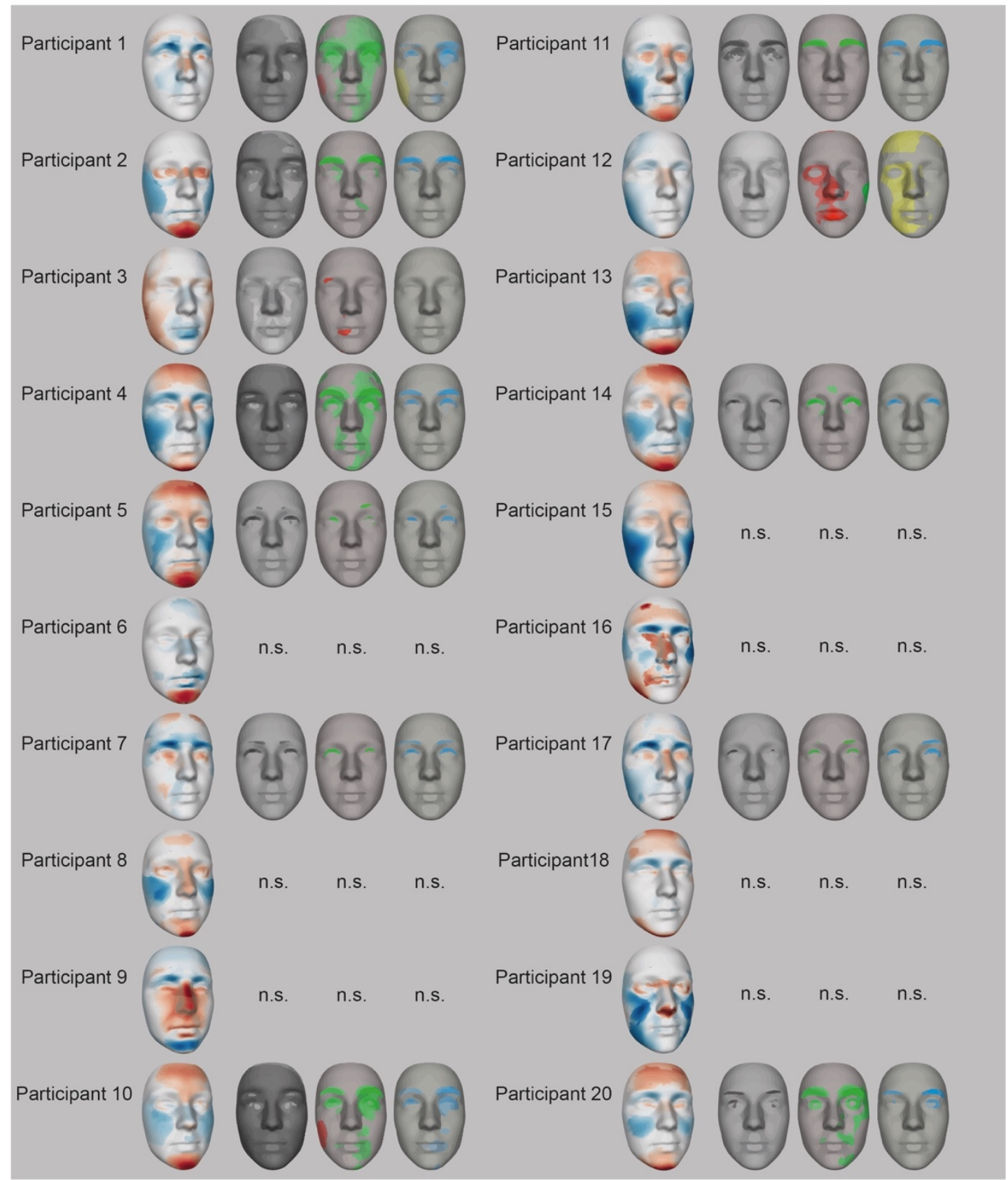

\section{B. Group Model}

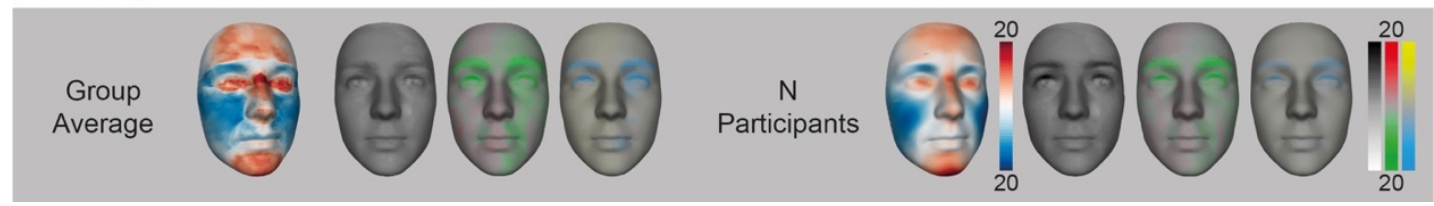

Fig. S2-3. A. Individual models of Eastern-other. B. Group model of Eastern-other. Same caption as Figure S2-1. 


\section{A. Individual Models}

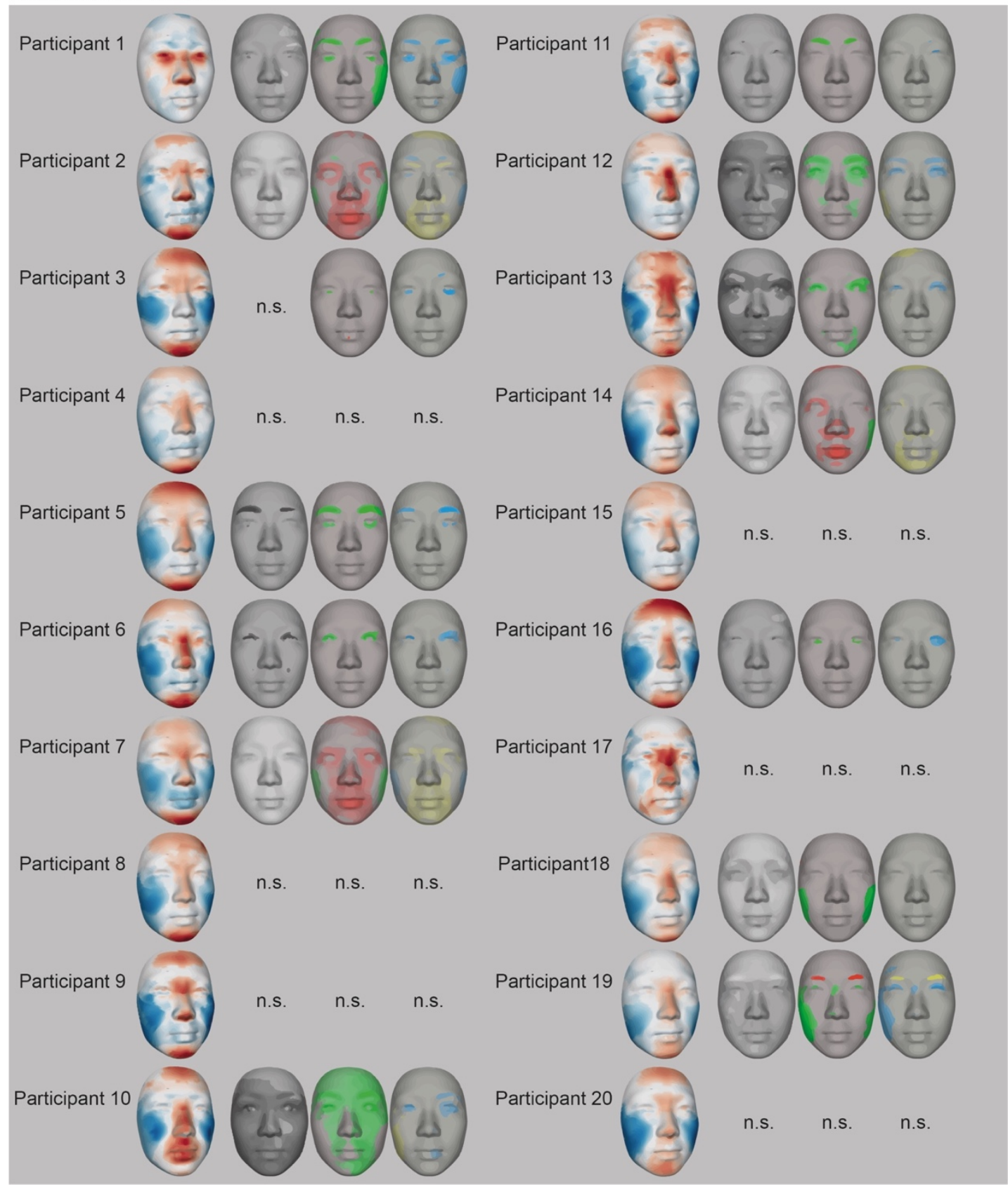

\section{B. Group Model}

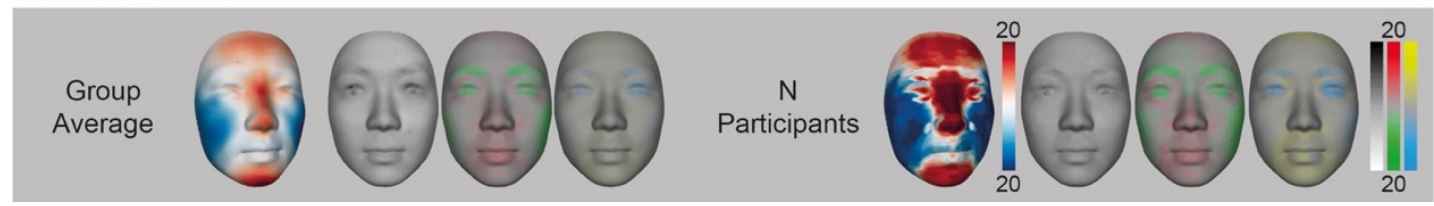

Fig. S2-4. A. Individual models of Eastern-same. B. Group model of Eastern-same. Same caption as Figure S2-1. 


\section{Features Dimensions WE-same Literatures}

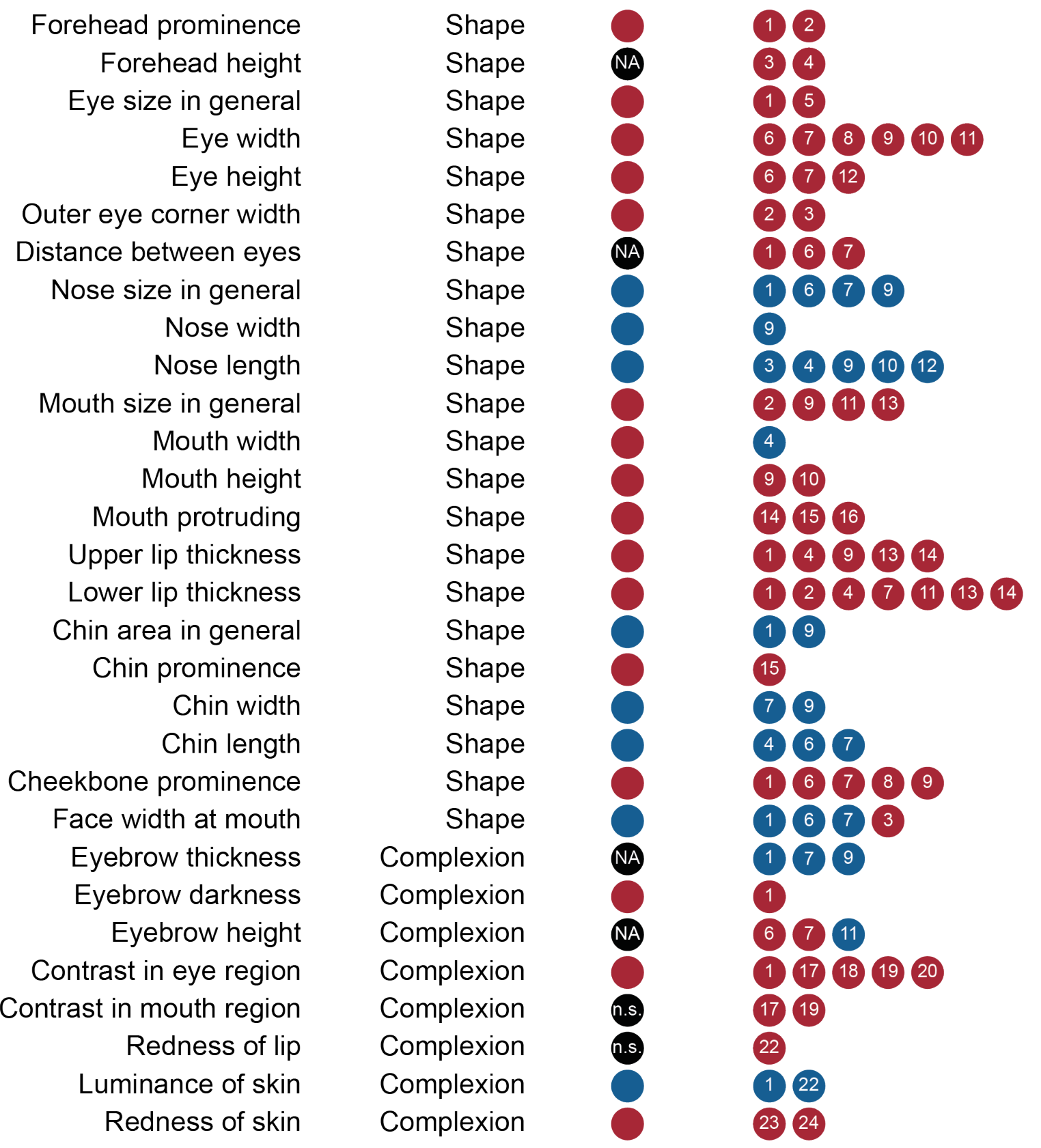

Fig. S3. Western-same (WE-same) model is consistent with the attractive features reported in the literature. Red (vs. blue) color denotes the positive (vs. negative) changes of the features in relation to the increased attractiveness. NA $=$ not applied in our model. n.s. $=$ not significant. Number $=$ SI reference index. 


\section{Features Dimensions EA-same Literatures}

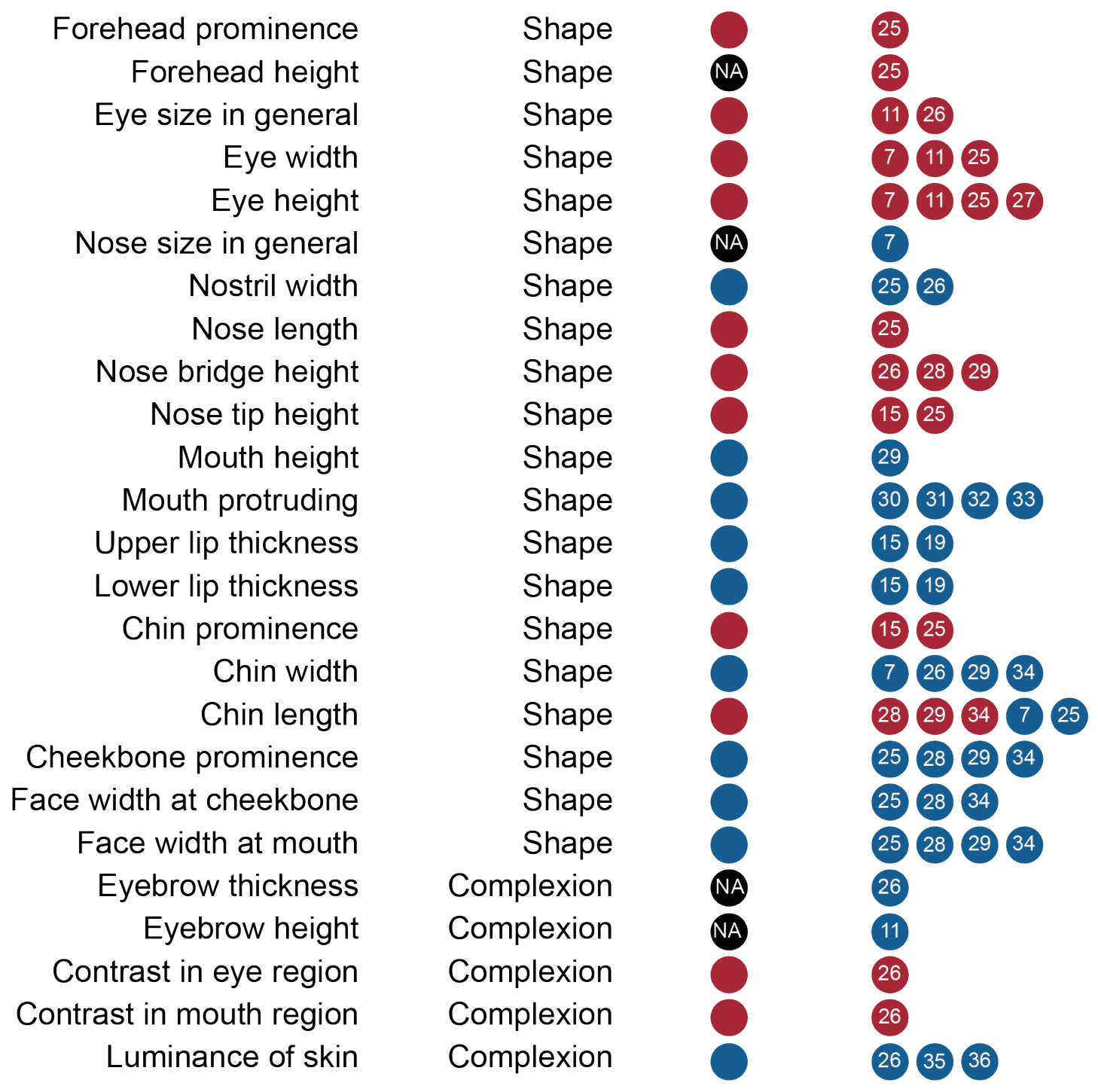

Fig. S4. Eastern-same (EA-same) model is consistent with the attractive features reported in the literature. Same caption as Figure S3. 

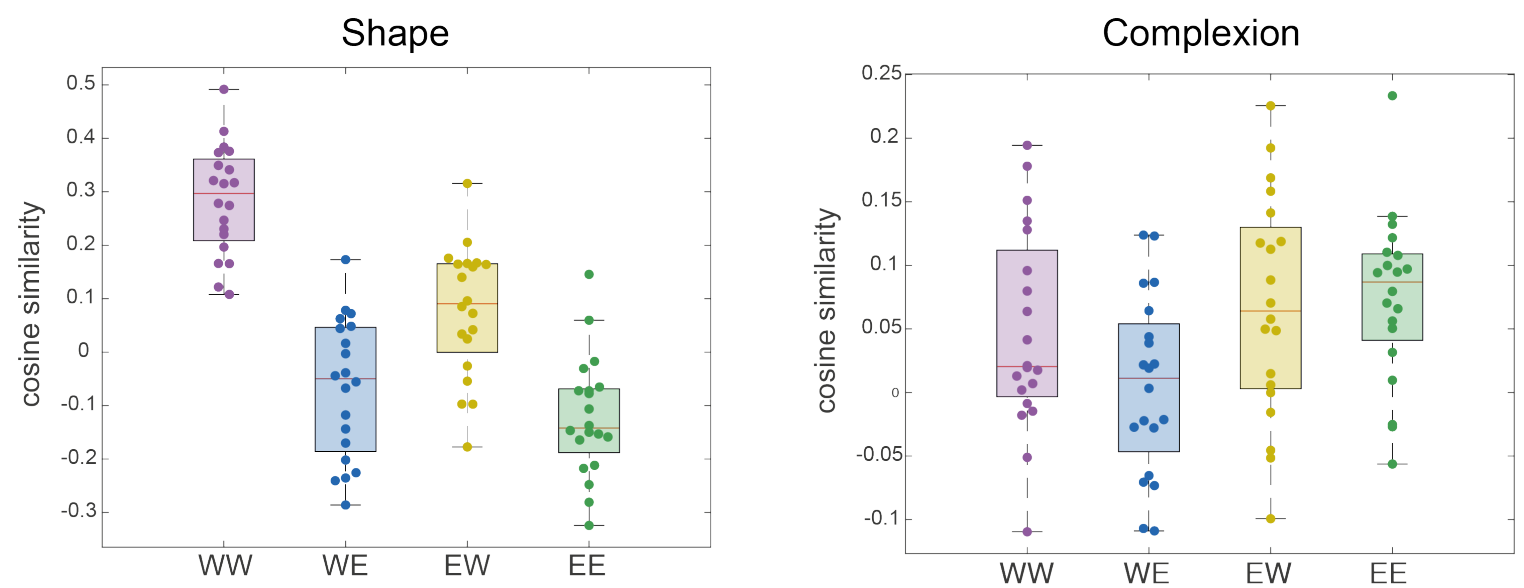

Fig. S5. Cosine similarity between sexual dimorphism and individual face attractiveness of shape (left panel) and complexion (right panel). Boxplots, color-coded by condition, show the cosine similarity of each individual model (overlaid as solid dots). WW is Western-same; WE, Westernother. EW, Eastern-other; EE, Eastern-same. 


\section{A. Attractive Components of Shape}
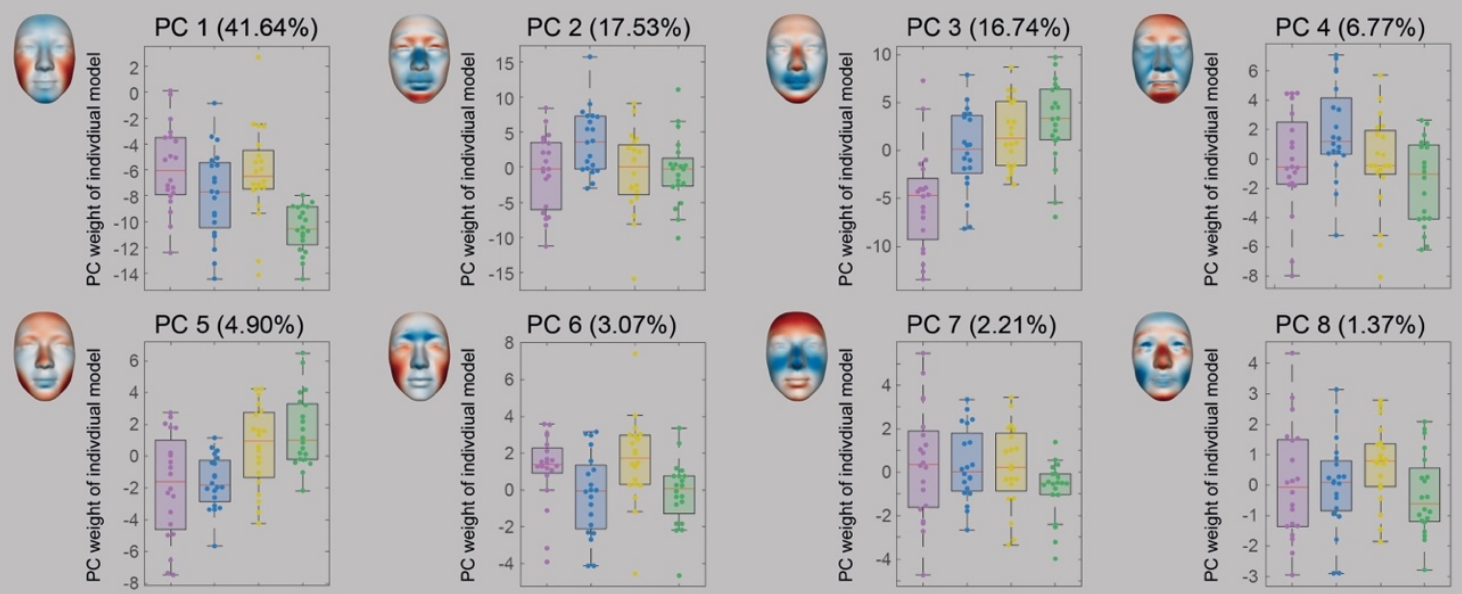

\section{B. Attractive Components of Complexion}
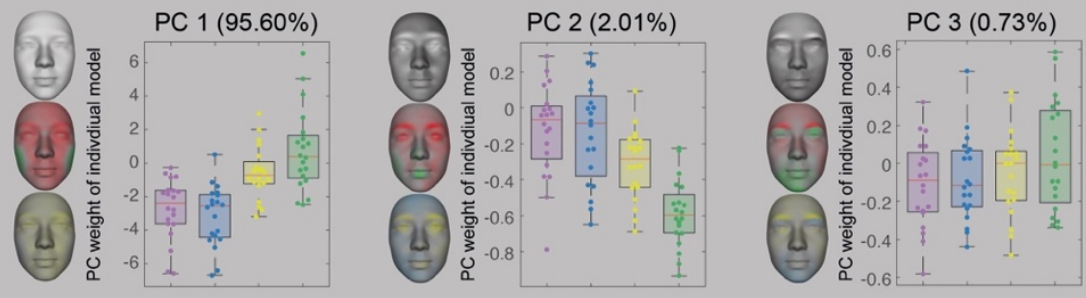

Western-same

- Western-other

Eastern-other

Eastern-same

Fig. S6. A. Attractive Components of Shape. In each panel, boxplots, color-coded by condition ( $x-$ axis), shows the weight of each individual model (y-axis) along each PC. Face maps illustrate the 3D shape deviations with positive weights. Bracketed percentage indicates the variance explained by the PC. B. Attractive Component of Complexion, displayed in the same format as A. 


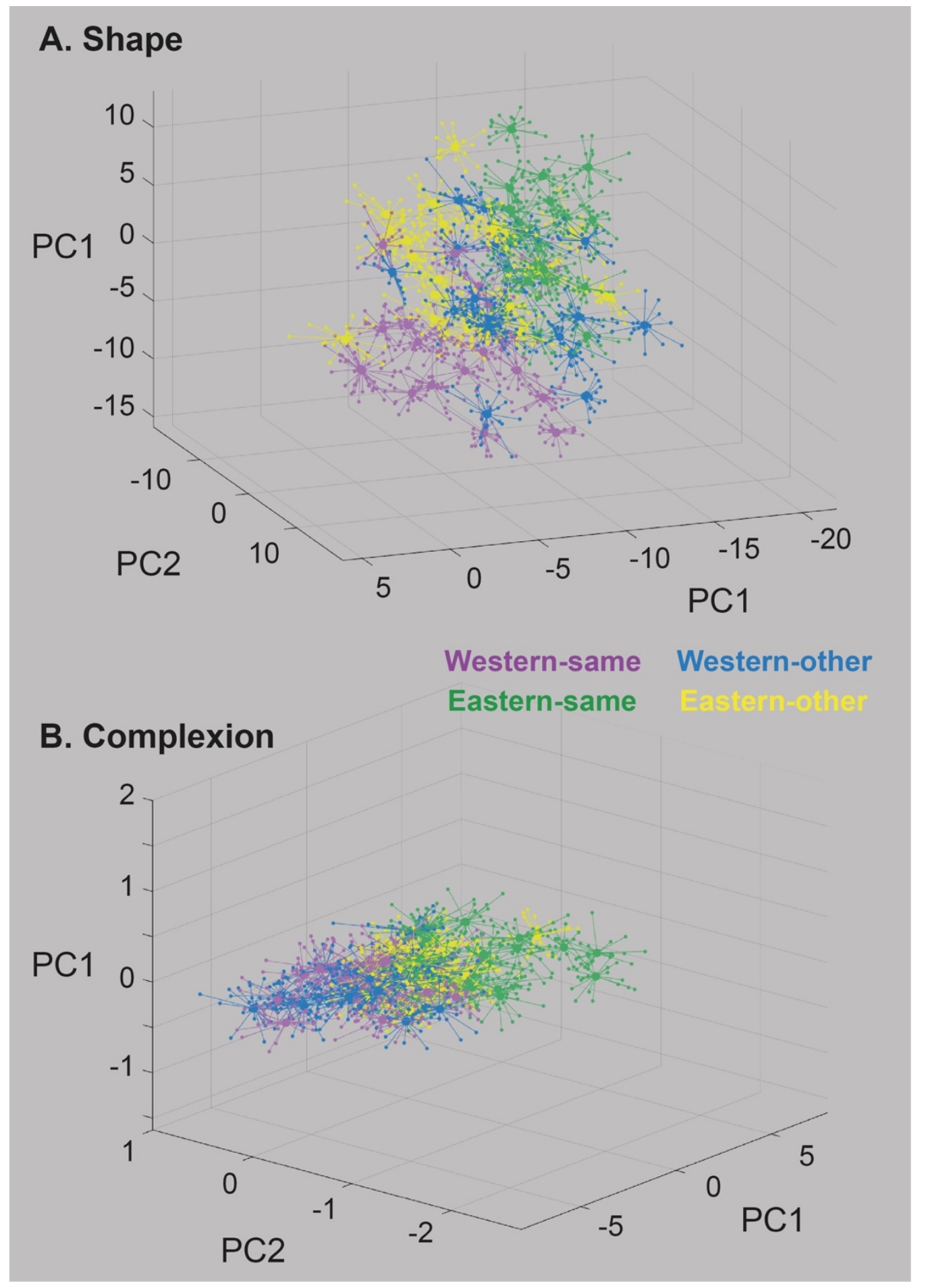

Fig. S7. A Shape attractiveness. In the representational space, each axis is a multivariate shape component of a principal components analysis that captures the variance of 1600 bootstrapped models of attractive face shape. Smaller colored dots represent 20 bootstrapped models per participant connected with a line to their average represented by the larger dots. B Complexion attractiveness, displayed in the same format as A. Purple represents Western-same, blue for Western-other, green for Eastern-same, and yellow for Eastern-other. 

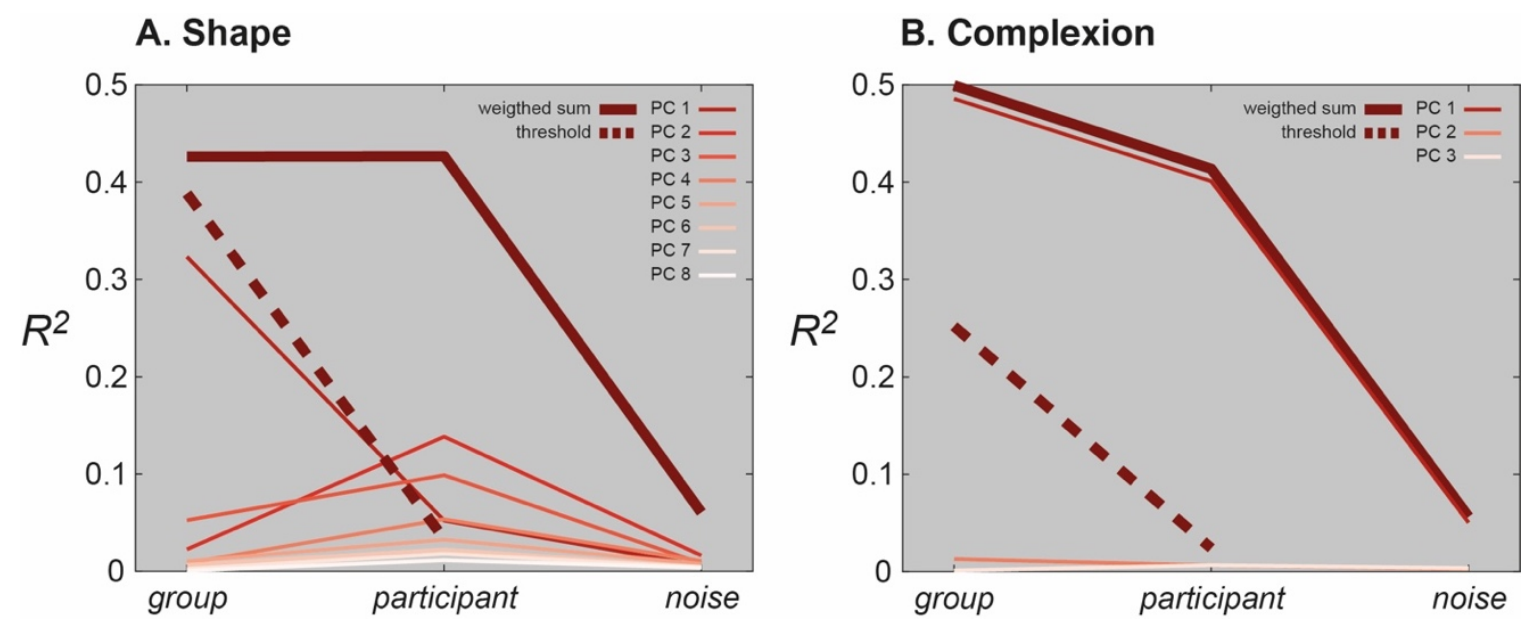

Figure S8. A Shape. Preference variance of shape attractiveness components (quantified by $R^{2}$ ), explained by 4 group averages (i.e. $R_{\text {group }}^{2}$ ), participants' idiosyncrasy (i.e. $R^{2}$ participant), plus the remaining unexplained variance (i.e. noise). Eight thin lines show the three decomposed $R^{2}$ for each of eight shape PCs, where color hues denote PC identities. Bold line (solid) shows the weighted sum of each $R^{2}$ over 8 PCs, scaled by the total variance explained by each PC; bold line (dash) illustrates their corresponding threshold based on a permutation test $(p<0.05)$. B Complexion, same as A, created for 3 complexion attractiveness components. 


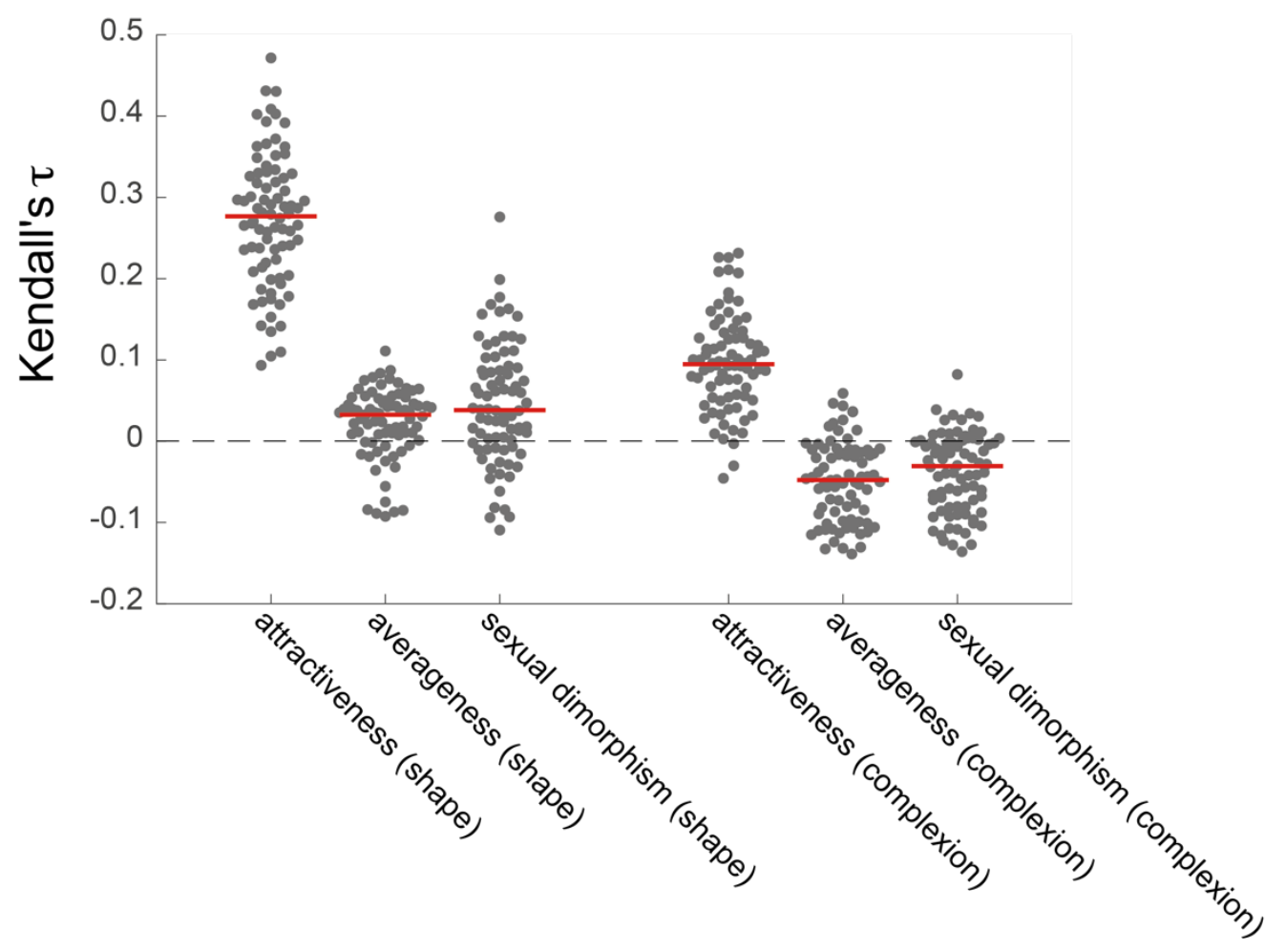

Fig. S9. Prediction performance (y-axis) of each tested GLM model (x-axis), measured by the Kendall's $\tau$ correlation between each participant's actual ratings vs. GLM predicted ratings. Red lines denote models' median prediction performance across 80 participants. 
A. Elbow Method

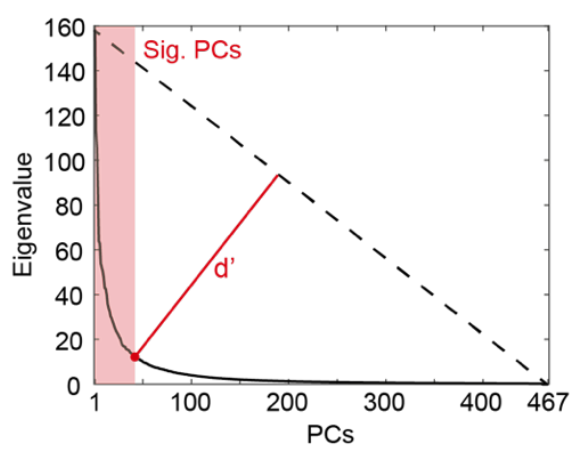

B. Sexual Dimorphism

Full GMF PCs

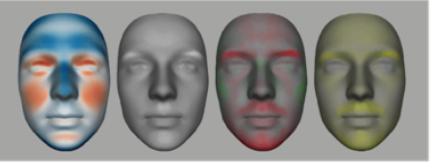

Sig. GMF PCs

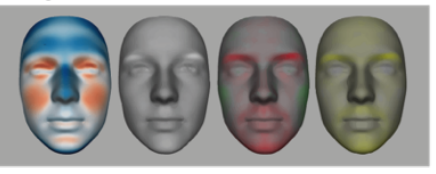

C. Attractiveness

Full Attractiveness PCs

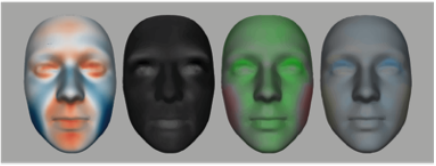

Sig. Attractiveness PCs

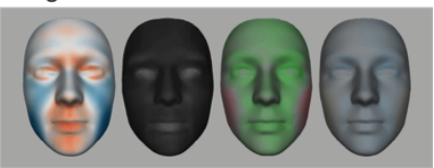

Fig. S10. A. Elbow Method. The elbow point (red point) on the Eigenvalue curve (black curve) has the largest distance (d') to the dash line connecting the first and last PCs. PCs explained significant variance are those before the elbow point. B. Sexual Dimorphism. Comparison of Full GMF PCs' representation (first row) and the representation of the significant PCs determined by Elbow Method (second row), separately for shape and $L^{*} a^{*} b^{*}$ complexion. C. Attractiveness, same as A. GMF = Generative Model of 3D Face. Sig. = Significant. 


\section{SI References}

1. C. Braun, M. Gründl, C. Marberger, C. Scherber, Beautycheck - causes and consequences of attractiveness. (2001).

2. C. Sforza, et al., Soft-Tissue Facial Characteristics of Attractive Italian Women as Compared to Normal Women. Angle Orthod 79, 17-23 (2009).

3. V. F. Ferrario, C. Sforza, C. E. Poggio, G. Tartaglia, Facial morphometry of television actresses compared with normal women. Journal of Oral and Maxillofacial Surgery 53, 1008-1014 (1995).

4. V. S. Johnston, M. Franklin, Is beauty in the eye of the beholder? Ethology and Sociobiology 14, 183-199 (1993).

5. S. Geldart, D. Maurer, K. Carney, Effects of Eye Size on Adults' Aesthetic Ratings of Faces and 5Month-Olds' Looking Times. Perception 28, 361-374 (1999).

6. M. R. Cunningham, Measuring the Physical in Physical Attractiveness: Quasi-Experiments on the Sociobiology of Female Facial Beauty. Journal of Personality and Social Psychology 50, 925-935 (1986).

7. M. R. Cunningham, A. R. Roberts, A. P. Barbee, P. B. Druen, C.-H. Wu, "Their ideas of beauty are, on the whole, the same as ours": Consistency and variability in the cross-cultural perception of female physical attractiveness. Journal of Personality and Social Psychology 68, 261-279 (1995).

8. K. Grammer, R. Thornhill, Human (Homo sapiens) facial attractiveness and sexual selection: The role of symmetry and averageness. Journal of Comparative Psychology 108, 233-242 (1994).

9. J.-Y. Baudouin, G. Tiberghien, Symmetry, averageness, and feature size in the facial attractiveness of women. Acta Psychologica 117, 313-332 (2004).

10. D. Jones, et al., Sexual Selection, Physical Attractiveness, and Facial Neoteny: Cross-cultural Evidence and Implications [and Comments and Reply]. Current Anthropology 36, 723-748 (1995).

11. S. C. Rhee, K.-S. Woo, B. Kwon, Biometric Study of Eyelid Shape and Dimensions of Different Races with References to Beauty. Aesth Plast Surg 36, 1236-1245 (2012).

12. L. Z. McArthur, K. Apatow, Impressions of Baby-Faced Adults. Social Cognition 2, 315-342 (1984).

13. M. Bisson, A. Grobbelaar, The Esthetic Properties of Lips: A Comparison of Models and Nonmodels. Angle Orthod 74, 162-166 (2004).

14. T. A. Auger, P. K. Turley, The female soft tissue profile as presented in fashion magazines during the 1900s: a photographic analysis. Int J Adult Orthodon Orthognath Surg 14, 7-18 (1999).

15. H. S. Oh, et al., Correlations between cephalometric and photographic measurements of facial attractiveness in Chinese and US patients after orthodontic treatment. American Journal of Orthodontics and Dentofacial Orthopedics 136, 762.e1-762.e14 (2009).

16. S. Matoula, H. Pancherz, Skeletofacial Morphology of Attractive and Nonattractive Faces. Angle Orthod 76, 204-210 (2006).

17. R. Russell, Sex, Beauty, and the Relative Luminance of Facial Features. Perception 32, 1093-1107 (2003).

18. R. Mulhern, G. Fieldman, T. Hussey, J.-L. Lévêque, P. Pineau, Do cosmetics enhance female Caucasian facial attractiveness? International Journal of Cosmetic Science 25, 199-205 (2003).

19. N. L. Etcoff, S. Stock, L. E. Haley, S. A. Vickery, D. M. House, Cosmetics as a Feature of the Extended Human Phenotype: Modulation of the Perception of Biologically Important Facial Signals. PLOS ONE 6, e25656 (2011).

20. D. Peshek, N. Semmaknejad, D. Hoffman, P. Foley, Preliminary Evidence that the Limbal Ring Influences Facial Attractiveness. Evol Psychol 9, 147470491100900200 (2011).

21. I. D. Stephen, A. M. McKeegan, Lip Colour Affects Perceived Sex Typicality and Attractiveness of Human Faces. Perception 39, 1104-1110 (2010).

22. A. G. Miller, W. A. Ashton, J. W. McHoskey, J. Gimbel, What Price Attractiveness? Stereotype and Risk Factors in Suntanning Behavior. Journal of Applied Social Psychology 20, 1272-1300 (1990).

23. B. Fink, K. Grammer, R. Thornhill, Human (Homo sapiens) facial attractiveness in relation to skin texture and color. Journal of Comparative Psychology 115, 92-99 (2001). 
24. A. D. Pazda, C. A. Thorstenson, A. J. Elliot, D. I. Perrett, Women's Facial Redness Increases Their Perceived Attractiveness: Mediation Through Perceived Healthiness. Perception 45, 739-754 (2016).

25. K. S. Jang, et al., A three-dimensional photogrammetric analysis of the facial esthetics of the Miss Korea pageant contestants. Korean J Orthod 47, 87 (2017).

26. K. Nakamura, K. Watanabe, Data-driven mathematical model of East-Asian facial attractiveness: the relative contributions of shape and reflectance to attractiveness judgements. $R$. Soc. open sci. 6, 182189 (2019).

27. L. Z. McArthur, D. S. Berry, Cross-Cultural Agreement in Perceptions of Babyfaced Adults. Journal of Cross-Cultural Psychology 18, 165-192 (1987).

28. Q. Zhao, et al., Morphological Quantitative Criteria and Aesthetic Evaluation of Eight Female Han Face Types. Aesth Plast Surg 37, 445-453 (2013).

29. S. Samizadeh, W. Wu, Ideals of Facial Beauty Amongst the Chinese Population: Results from a Large National Survey. Aesth Plast Surg 42, 1540-1550 (2018).

30. J. Soh, M. T. Chew, H. B. Wong, An Asian community's perspective on facial profile attractiveness. Community Dentistry and Oral Epidemiology 35, 18-24 (2007).

31. J. Soh, M. T. Chew, H. B. Wong, Professional assessment of facial profile attractiveness. American Journal of Orthodontics and Dentofacial Orthopedics 128, 201-205 (2005).

32. H. Ioi, T. Shimomura, S. Nakata, A. Nakasima, A. L. Counts, Comparison of anteroposterior lip positions of the most-favored facial profiles of Korean and Japanese people. American Journal of Orthodontics and Dentofacial Orthopedics 134, 490-495 (2008).

33. S. Kuroda, et al., Influence of anteroposterior mandibular positions on facial attractiveness in Japanese adults. American Journal of Orthodontics and Dentofacial Orthopedics 135, 73-78 (2009).

34. S. C. Rhee, S. H. Lee, Attractive Composite Faces of Different Races. Aesth Plast Surg 34, 800-801 (2010).

35. B. J. Dixson, A. F. Dixson, B. Li, M. J. Anderson, Studies of human physique and sexual attractiveness: Sexual preferences of men and women in China. American Journal of Human Biology 19, 88-95 (2007).

36. C. Han, et al., Cultural differences in preferences for facial coloration. Evolution and Human Behavior 39, 154-159 (2018). 